\title{
The interaction of DiaA and DnaA regulates the replication cycle in $E$. coli by directly promoting ATP-DnaA-specific initiation complexes
}

\author{
Kenji Keyamura, ${ }^{1,5}$ Norie Fujikawa, ${ }^{2,5}$ Takuma Ishida, ${ }^{1}$ Shogo Ozaki, ${ }^{1}$ Masayuki Su'etsugu, ${ }^{1}$ \\ Kazuyuki Fujimitsu, ${ }^{1}$ Wataru Kagawa, ${ }^{2}$ Shigeyuki Yokoyama, ${ }^{2,3}$ Hitoshi Kurumizaka, ${ }^{2,4,7}$ \\ and Tsutomu Katayama ${ }^{1,6}$ \\ ${ }^{1}$ Department of Molecular Biology, Graduate School of Pharmaceutical Sciences, Kyushu University, Fukuoka 812-8582, \\ Japan; ${ }^{2}$ RIKEN Genomic Sciences Center, 1-7-22 Suehiro-cho, Tsurumi, Yokohama 230-0045, Japan; ${ }^{3}$ Department of \\ Biophysics and Biochemistry, Graduate School of Science, University of Tokyo, 7-3-1 Hongo, Bunkyo-ku, Tokyo 113-0033, \\ Japan; ${ }^{4}$ Graduate School of Science and Engineering, Waseda University, 3-4-1 Okubo, Shinjuku-ku, Tokyo 169-8555, Japan
}

Escherichia coli DiaA is a DnaA-binding protein that is required for the timely initiation of chromosomal replication during the cell cycle. In this study, we determined the crystal structure of $\mathrm{DiaA}$ at $1.8 \AA$ resolution. DiaA forms a homotetramer consisting of a symmetrical pair of homodimers. Mutational analysis revealed that the DnaA-binding activity and formation of homotetramers are required for the stimulation of initiation by DiaA. DiaA tetramers can bind multiple DnaA molecules simultaneously. DiaA stimulated the assembly of multiple DnaA molecules on oriC, conformational changes in ATP-DnaA-specific initiation complexes, and unwinding of oriC duplex DNA. The mutant DiaA proteins are defective in these stimulations. DiaA associated also with ADP-DnaA, and stimulated the assembly of inactive ADP-DnaAoriC complexes. Specific residues in the putative phosphosugar-binding motif of DiaA were required for the stimulation of initiation and formation of ATP-DnaA-specific-oriC complexes. Our data indicate that DiaA regulates initiation by a novel mechanism, in which DiaA tetramers most likely bind to multiple DnaA molecules and stimulate the assembly of specific ATP-DnaA-oriC complexes. These results suggest an essential role for DiaA in the promotion of replication initiation in a cell cycle coordinated manner.

[Keywords: initiation of replication; protein complex; complex structure; DNA dynamics; cell cycle regulation; initiator regulation]

Supplemental material is available at http://www.genesdev.org.

Received April 12, 2007; revised version accepted July 9, 2007.

In prokaryotes and eukaryotes, the initiation of chromosomal replication is strictly regulated and occurs at a specific time during the cell cycle (Messer 2002; Stillman 2005; Kaguni 2006; Botchan 2007). A crucial aspect of replication initiation is the ordered assembly and conformational change of specific proteins at the replication origin. In Escherichia coli, multiple ATP-bound DnaA (ATP-DnaA) molecules form active complexes with the origin of replication (oriC) and unwind the duplex DNA within oriC, resulting in replication initiation. DnaA monomers specifically bind to sequences within oriC called DnaA boxes and interact with other DnaA mol-

\footnotetext{
${ }^{5}$ These authors contributed equally to this work.

Corresponding authors.

${ }^{6}$ E-MAIL katayama@phar.kyushu-u.ac.jp; FAX 81-92-642-6646.

${ }^{7}$ E-MAIL kurumizaka@waseda.jp; FAX 81-3-5292-9211.

Article is online at http://www.genesdev.org/cgi/doi/10.1101/gad.1561207.
}

ecules. These activities potentiate the formation of DnaA homomultimers on oriC. The present study is the first demonstration that DiaA, a DnaA-binding protein (Ishida et al. 2004), forms homotetramers, directly stimulates assembly of multiple ATP-DnaA molecules on oriC, and positively regulates a conformational change of the DnaA multimer-oriC complex to an initiation-competent state.

A single E. coli cell contains $\sim 1000$ molecules of the initiator protein DnaA, which exists in either an ATPDnaA active form or an ADP-bound inactive form (ADPDnaA) (Messer 2002; Kaguni 2006). The level of ATPDnaA in the cell temporarily increases during the cell cycle at the same time as the initiation of replication (Kurokawa et al. 1999). The E. coli oriC contains several DnaA-binding sites, including several repeats of a 9-mer sequence called the DnaA box. When ATP-DnaA forms a multimeric complex on oriC, the DNA duplex is un- 
wound specifically at 13-mer AT-rich repeats within oriC (Speck and Messer 2001; McGarry et al. 2004; Kawakami et al. 2005). DnaB helicase is then loaded onto the unwound strands of DNA, and the single-stranded region is extended. DnaG primase and DNA polymerase (Pol) III holoenzyme then assemble on the singlestranded DNA to replicate the complementary strand. After the completion of Okazaki fragments, the $\beta$-clamp, which is a component of Pol III holoenzyme, remains on the nascent DNA strand (O'Donnell 2006). Hda forms a complex with the DNA-loaded $\beta$-clamp, which promotes hydrolysis of DnaA-ATP to ADP-DnaA (Katayama et al. 1998; Kato and Katayama 2001). This process for inactivating DnaA, termed the regulatory inactivation of DnaA (RIDA), is required for repressing untimely or excessive initiation events.

The timing of replication initiation is tightly coupled with cell cycle progression (Zyskind and Smith 1992). In E. coli, when the growth rate of cells is rapid, a single cell contains replication intermediates on multiple chromosomes, and replication initiates on each chromosome at exactly the same time. This level of regulation implies that there are multiple mechanisms in place to ensure that replication initiation is coupled to specific cell cycle events, that ATP-DnaA molecules accumulate to the proper level, and that the formation of ATP-DnaA-oriC complexes occurs in a timely manner. These mechanisms of regulation have yet to be fully explored.

In eukaryotes, initiation of DNA replication requires many proteins that directly or indirectly interact with the replication origin (Stillman 2005; Botchan 2007). In Saccharomyces cerevisiae, the origin recognition complex (ORC), a nucleoprotein complex that binds to the origin of replication, is preserved during the cell cycle. A pre-replication complex is formed when the MCM helicase complex is loaded onto the ORC-DNA complex, which also contains Cdc6 and Cdt1. The initiation of replication is activated by cyclin-dependent kinase at the $\mathrm{G}_{1} / \mathrm{S}$ transition, and several proteins, including Cdc 45 , the 11-3-2 complex, and the GINS complex are loaded onto the pre-replication complex to form an initiationcompetent replication complex in a timely manner.

In E. coli, DiaA is required to ensure the timely initiation of chromosomal replication during the cell cycle (Ishida et al. 2004). The diaA gene was first identified in a search for intergenic suppressor mutations of the $d n a A$ mutant dnaAcos, in which there is overinitiation of chromosomal replication, and colony formation at $30^{\circ} \mathrm{C}$ is inhibited. Replication initiation in the dnaAcos mutant is resistant to RIDA at this temperature (Katayama and Crooke 1995). In diaA gene-disrupted cells, initiation is retarded, and the synchronous initiation of replication on multiple chromosomes at oriC is disrupted (Ishida et al. 2004). In the diaA mutant, when two oriC copies are present in a single, rapidly growing cell, only one oriC initiates, which produces a cell bearing three copies of oriC. In wild-type cells, this is an extremely rare event. Mutation of diaA also inhibits the stable maintenance of minichromosomes and, in a dnaA46temperature sensitive mutant, causes synthetic lethality at $37^{\circ} \mathrm{C}$. Thus, DiaA is a positive regulator to stimulate the initiation. This function of DiaA is independent of RIDA and SeqA, an oriC-binding protein that represses extra initiations (Slater et al. 1995). Purified DiaA directly and specifically binds to DnaA, independent of the nucleotide- or DNA-bound state of DnaA (Ishida et al. 2004). DiaA binds to domains I-II in the N terminus of DnaA. The overall primary sequence of DiaA indicates that it contains a sugar isomerase (SIS) domain (Bateman 1999|. This domain is proposed to be a consensus site for binding phosphosugars and is found in several proteins, such as the glucokinase regulator protein.

In the present study, we isolated several plasmid-encoded dysfunctional diaA mutants using complementation analysis of the dnaAcos diaA::Tn5 mutant. In parallel, we determined the crystal structure of DiaA at 1.8 $\AA$ resolution. The structure revealed that DiaA forms a homotetramer consisting of a symmetrical pair of homodimers. Analysis of mutant DiaA proteins identified residues specifically required for DnaA binding. We also identified specific sites located in the interdimer interface that were required for homotetramer formation. Another group of mutations was located in the putative phosphosugar-binding region. All the DiaA mutants were inactive in stimulating replication initiation.

We also showed that a single DiaA tetramer can bind multiple DnaA molecules simultaneously. DiaA tetramers stimulated the assembly of multiple DnaA molecules on oriC, ATP-DnaA-specific conformational changes of the initiation complex, and unwinding of the duplex DNA at oriC. Mutant DiaA proteins were defective in all these activities. We propose that DiaA regulates initiation via a novel mechanism, in which a single DiaA tetramer binds to multiple DnaA molecules to stimulate inter-DnaA interactions, resulting in efficient assembly of multiple ATP-DnaA molecules on oriC and stimulation of initiation complex formation. This unique role for DiaA is most likely essential for the promotion of initiation in a cell cycle coordinated manner.

\section{Results}

\section{Isolation of dysfunctional diaA mutations}

To determine the functional mechanism of DiaA-mediated stimulation of replication initiation, we isolated diaA mutant alleles using in vitro Mn-PCR mutagenesis and in vivo complementation analysis. The plasmid pTKM601 encodes a histidine (His) fusion of wild-type diaA downstream from the arabinose-inducible promoter of pBAD/HisB. The dnaAcos diaA26::Tn5 double mutant (NA26) grew at $30^{\circ} \mathrm{C}$, but colony-forming ability at this temperature was inhibited in the presence of pTKM601 and $0.05 \%$ arabinose (Table 1). These results indicated that His-tagged DiaA (hisDiaA) was functional in the stimulation of chromosomal replication. We also introduced several pTKM601 derivatives bearing randomly mutagenized diaA alleles into NA26 cells. The mutant diaA alleles were unable to complement the diaA26::Tn5 allele, and the transformants formed colo- 
Table 1. DiaA complementation test

\begin{tabular}{|c|c|c|c|c|}
\hline \multirow[b]{3}{*}{ Strain } & \multirow[b]{3}{*}{ Plasmid } & \multicolumn{2}{|c|}{ Arabinose } & \multirow[b]{2}{*}{ Ratio } \\
\hline & & $0.05 \%$ & $0 \%$ & \\
\hline & & \multicolumn{2}{|c|}{$\left(\times 10^{9}\right) \mathrm{CFU}^{\mathrm{a}} / \mathrm{mL}$} & $0.05 \% / 0 \%$ \\
\hline KH5402-1 (wild type) & pBAD/HisB (Vector) & 3.0 & 3.5 & 0.86 \\
\hline NA26 (dnaAcos diaA26::Tn5) & $\mathrm{pBAD} / \mathrm{HisB}$ & 3.1 & 3.1 & 1.0 \\
\hline & pTKM601 (wild-type diaA) & $6.0 \times 10^{-6}$ & 1.6 & $3.8 \times 10^{-6}$ \\
\hline & pMn17 (diaA L23P) & 4.4 & 3.7 & 1.2 \\
\hline & $\mathrm{pMn} 20($ diaA $L 45 P)$ & 3.2 & 3.3 & 0.97 \\
\hline & pMn53 (diaA S52F) & 2.9 & 3.2 & 0.91 \\
\hline & pMn18 (diaA A53T) & 3.1 & 3.7 & 0.84 \\
\hline & pMn52 (diaA R71Q) & 3.1 & 3.8 & 0.82 \\
\hline & pMn31 (diaA L79P) & 2.7 & 3.6 & 0.75 \\
\hline & pMn57 (diaA N83D) & 1.4 & 2.4 & 0.58 \\
\hline & pMn30 (diaA K101E) & 2.4 & 2.2 & 1.1 \\
\hline & pMn51 (diaA E173G) & 3.4 & 2.9 & 1.2 \\
\hline & pMn6 (diaA L177P) & 2.2 & 2.1 & 1.0 \\
\hline & pMn14 (diaA C183Y) & 3.7 & 3.1 & 1.2 \\
\hline & pMn28 (diaA L190P) & 4.1 & 3.7 & 1.1 \\
\hline & pMn29 (diaA P72S V85A) & 3.5 & 3.1 & 1.1 \\
\hline & pMn21 (diaA E173G F191S) & 4.8 & 4.4 & 1.1 \\
\hline & pMn54 (diaA A56V F191L) & 3.2 & 3.0 & 1.1 \\
\hline & pMn48 (diaA S28P S165P) & 3.4 & 3.3 & 1.0 \\
\hline & pMn72 (diaA S37T N65D) & 2.5 & 4.0 & 0.63 \\
\hline & pMn61 (diaA M63K R93H) & 3.9 & 4.3 & 0.91 \\
\hline & pMn44 (diaA S73R E97G) & 3.4 & 2.8 & 1.2 \\
\hline & pMn40 (diaA D96G L182P) & 4.1 & 4.0 & 1.0 \\
\hline & pMn27 (diaA F9S A31T L39P) & 3.4 & 3.0 & 1.1 \\
\hline & pMn13 (diaA N80Y I116V T178A) & 3.2 & 3.6 & 0.89 \\
\hline & pdiaA $P 72 A$ & 2.6 & 2.6 & 1.0 \\
\hline & pdiaA P72S & 2.3 & 2.5 & 1.1 \\
\hline & pdiaA L190A & 2.6 & 2.6 & 1.0 \\
\hline & pdiaA F191L & 0.43 & 0.27 & 0.63 \\
\hline & pdiaA F191S & 0.70 & 0.68 & 0.97 \\
\hline
\end{tabular}

Cells of each strain were grown overnight at $30^{\circ} \mathrm{C}$, plated on LB plates containing $0.05 \%$ or $0 \%$ arabinose, and incubated for $19 \mathrm{~h}$ at $30^{\circ} \mathrm{C}$. Ampicillin and thymine were also included in media.

${ }^{\mathrm{a} C}$ Colony forming units.

nies at $30^{\circ} \mathrm{C}$ in the presence of $0.05 \%$ arabinose. We isolated 86 independent transformants and found that 26 of them overproduced a protein that was equivalent in size to full-length hisDiaA expressed in the presence of $0.5 \%$ arabinose (data not shown).

The mutant diaA alleles were analyzed by nucleotide sequencing (Table 1; Supplemental Table S1), which revealed that there were 22 distinct diaA mutants. Based on the sequences of pMn21, pMn29, and pMn54 (Table 1), plasmids encoding DiaA (pdiaA) that carried a single amino acid substitution of Pro72 or Phe191 were constructed by site-directed mutagenesis (for Pro72) or by restriction enzyme digestion and replacement (for Phe191). A plasmid encoding diaA bearing an alanine substitution of Leu190 (pdiaA L190A) was also constructed by site-directed mutagenesis, based on the sequence of pMn28 (diaA L190P). These plasmids were reintroduced into NA26 cells, and colony formation was assessed. As seen in Table 1, these mutant alleles were unable to complement the diaA-null allele in the growth inhibition of the dnaAcos mutant at $30^{\circ} \mathrm{C}$ and therefore are suggested to be impaired in the stimulation of chro- mosomal replication. The plasmids were also introduced into NA001 (dnaAcos) cells. No diaA mutant alleles except for only diaA L190P affected the cold sensitivity in colony formation (data not shown). DiaA L190P might inhibit the function of wild-type DiaA.

Classification of diaA mutations based on the crystal structure of DiaA

To determine the location of the diaA mutations in the DiaA structure, we resolved the crystal structure of DiaA at $1.8 \AA$ resolution (Table 2). The structure revealed a tetrameric DiaA molecule (Fig. 1B). A bundle of three parallel $\beta$-sheets that is wrapped by seven $\alpha$-helices forms a protomer. The two dimers form a symmetrical tetramer via a hydrogen-bond network in the core region formed by the $\alpha$-helices (Supplemental Fig. S1). Each protomer in the crystal structure is identical. A symmetrical dimer includes disulfide bonds between Cys8 and Cys181 on $\alpha$-helices of each protomer. A diaA mutant allele bearing an amino acid substitution of C181S 
Table 2. X-ray data collection, phasing, and refinement statistics

\begin{tabular}{ll}
\hline Data collection & BL44B2, SPring-8 \\
Beam line & 1.000 \\
Wavelength $(\AA)$ & $50-1.85(1.92-1.85)$ \\
Resolution $(\AA)$ (last shell) & 29,746 \\
Unique reflections & 4.0 \\
Redundancy & $99.9(99.9)$ \\
Completeness $(\%)($ last shell) & $18.5(8.0)$ \\
$I / \sigma(I)($ last shell) & $4.2(17.2)$ \\
$R_{\text {sym }}{ }^{(\%)}($ last shell) & \\
Refinement statistics & \\
Resolution $(\AA)$ & $50-1.85$ \\
$R_{\text {work }}(\%)$ & 18.3 \\
$R_{\text {free }}(\%)$ & 21.4 \\
Average $B$-factor $\left(\AA^{2}\right)$ & 18.1 \\
Minimum $B$-factor $\left(\AA^{2}\right)$ & 2.62 \\
Maximum $B$-factor $\left(\AA^{2}\right)$ & 61.3 \\
RMSD bond length $(\AA)$ & 0.006 \\
RMSD bond angles $\left({ }^{\circ}\right)$ & 1.24 \\
RMSD dihedrals $\left({ }^{\circ}\right)$ & 20.7 \\
RMSD impropers $\left({ }^{\circ}\right)$ & 0.702 \\
\hline
\end{tabular}

${ }^{\mathrm{a}} R_{\text {sym }}=\Sigma\left|I_{\text {avg }}-I i\right| / \Sigma I i$.

${ }^{\mathrm{b}} R_{\text {free }}$ is calculated for $10 \%$ of randomly selected reflections excluded from refinement.

complemented the diaA-null allele of NA26 (dnaAcos diaA::Tn5) cells in the cold-sensitive growth (data not shown; Supplemental Table S1), suggesting that the disulfide bonds are not required for the in vivo function (also see below).

The locations of several of the diaA mutations in the crystal structure (Fig. 1C) mapped to the protomer surface and were clustered in three structurally distinct regions. The first region, represented by Asn 83 and Lys101, was located at the core of the interdimer interface of tetrameric DiaA, which contains a network of hydrogen bonds that creates a "caged structure" (Fig. 1B,C; Supplemental Fig. S1). This structural network appeared to be essential for tetramer formation via the association of a pair of homodimers (see below).

The second region, represented by Arg71, Pro72, Leu190, and Phe191, was located at the outer edge of the DiaA surface (Fig. 1B,C). This region represents a potential interaction site with other proteins, such as DnaA. The third region, represented by Ser52 and Ala53, was located in the putative phosphosugar-binding region (Fig. 1B,C). Pseudomonas aeruginosa GmhA has an SIS domain and is highly homologous in sequence to DiaA (Fig. 1A; Brooke and Valvano 1996; Bateman 1999). The crystal structure of GmhA in complex with glycero-D-mannopyranose-7-phosphate was previously determined (Protein Data Bank ID 1X92) and shows that Ser54 of GmhA, which corresponds to DiaA Ser52, is located on phosphosugar-binding surface (Supplemental Fig. S2).

\section{Regulation of initiation of chromosomal replication} is defective in diaA mutants

To determine whether the diaA mutant alleles described above were functional in the regulation of chromosomal replication initiation, we performed flow cytometric analysis. Mutant diaA26::Tn5 cells bearing wild-type or diaA mutant alleles on a mini-R low-copy plasmid were grown to exponential phase at $30^{\circ} \mathrm{C}$ in $\mathrm{LB}$ medium in the presence or absence of $0.2 \%$ glucose, then allowed to incubate for an additional period of time in the presence of rifampicin and cephalexin to allow run-out replication, and then analyzed by flow cytometry (Fig. 2). We previously revealed by flow cytometry analysis that introduction of diaA-carrying pBR322 slightly inhibits the initiation of chromosomal replication but that of diaAcarrying mini-R plasmid does not (Ishida et al. 2004). In the present experiments, to avoid overproduction of DiaA, we used a mini-R low-copy vector. The mini-R plasmid used here is a derivative of the R 1 plasmid and bears the replication origin, the gene encoding the initiator RepA protein, the copy number-regulating inc regions, and the plasmid-partitioning par regions (Ishida et al. 2004; Nordström 2006). The copy number of this plasmid is strictly maintained at a level similar to that of the chromosome. After duplication, daughter plasmids are actively and evenly partitioned to two daughter cells.

In cells that expressed wild-type diaA, in the presence of glucose, there were two predominant peaks in the flow cytometry histogram, which indicated that four or eight chromosomes are present in these cells (Fig. 2A). In these same cells in the absence of glucose, there were predominantly four chromosomes (Fig. 2B). In these experiments, glucose was added to increase the growth rate of cells. We can observe the effect of DiaA in cells with different growth rates.

In contrast to the profiles of wild-type diaA-expressing cells (wild-type cells), in cells expressing mutant diaA alleles (mutant cells), the pattern of the peaks in the flow cytometry histogram demonstrated the presence of asynchronous initiation events (Fig. 2A,B). In $0.2 \%$ glucoseLB medium, the number of mutant cells carrying eight chromosomes was decreased compared to that of wildtype cells, which indicated that the timing of initiation in mutant cells is delayed, relative to the progression of the cell cycle (Fig. 2A). Inhibition of initiation was also observed in diaA mutant cells in LB medium lacking glucose (Fig. 2B). These results indicated that the stimulatory function of DiaA in replication initiation is defective in the mutant cells, and that at least eight amino acid residues-Ser52, Ala53, Arg71, Pro72, Asn83, Lys101, Leu190, and Phe191-are critical for the function of DiaA. The effect of the mutation P72S was limited in inhibition of initiation unlike that of P72A (Fig. 2A,B). Both DiaA P72S and DiaA P72A were clearly defective in the complementation test using the dnaAcos diaA-null mutant at $30^{\circ} \mathrm{C}$ (Table 1). Interaction of DiaA P72S with DnaAcos might be more severely inhibited than that with wild-type DnaA.

Immunoblot analysis showed that the amount of DiaA was similar in cells bearing mutant diaA alleles and wild-type cells, with the exception of cells expressing DiaA K101E, in which the level of DiaA was increased approximately twofold compared to wild-type DiaA when cells were grown in LB medium (Fig. 2C,D). These 
A

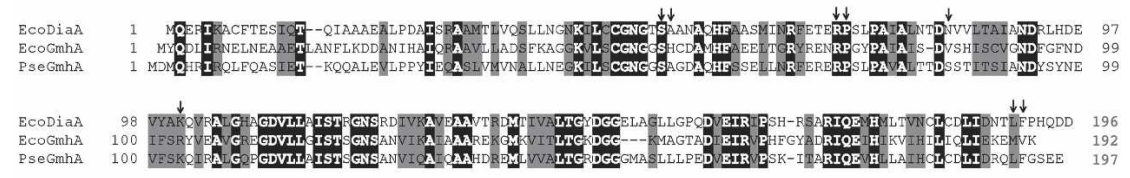

B
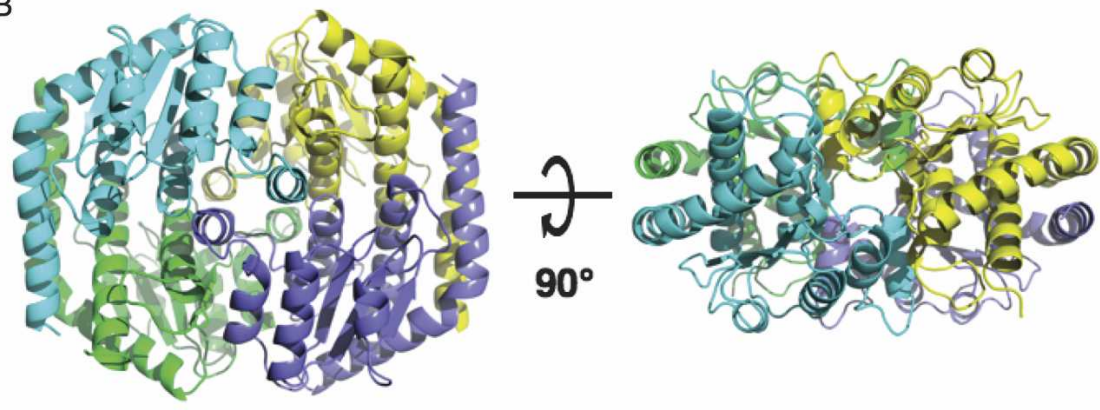

C

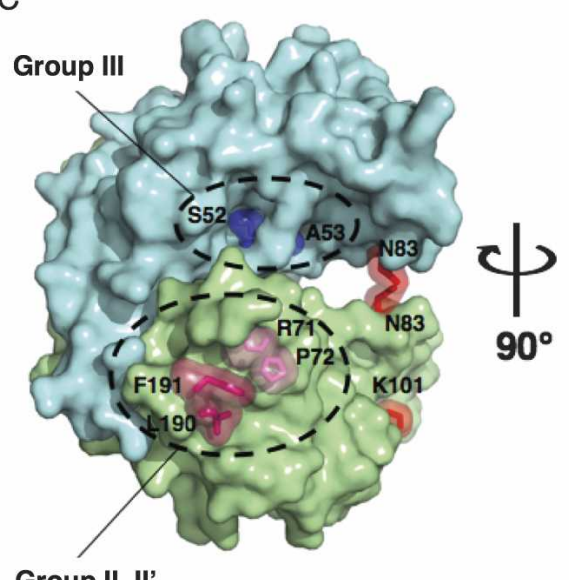

Group II, II'

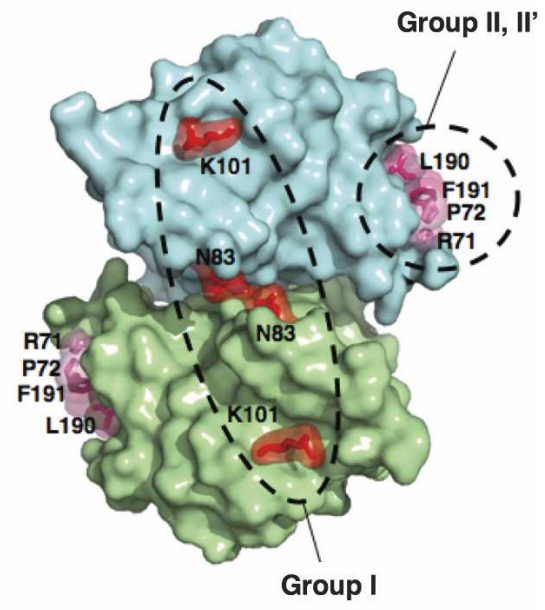

Figure 1. Structural features of E. coli DiaA. (A) Amino acid sequences of E. coli (Eco) DiaA, GmhA, and P. aeruginosa (Pse) $\mathrm{GmhA}$. Identical residues are highlighted in black, and chemically similar residues are shaded. Arrows indicate the DiaA residues mainly analyzed in this study. (B) Crystal structure of an EcoDiaA tetramer. Each protomer is depicted as a ribbon diagram in different colors. $(C)$ Location of the amino acid residues examined in this study on two EcoDiaA protomers. The two protomers correspond to the left half of the EcoDiaA tetramer depicted in $B$, left figure, and are rotated $30^{\circ}$ along the $X$-axis. Amino acids belonging to (red) Group I, (purple) Group II, (pink) Group II', and (blue) Group III (see text): Group I, the dimer-dimer interface; Group II, the sites essential for DnaA binding; Group II', the sites near the essential DnaA-binding site; and Group III, the putative phosphosugar-binding region. results indicated that the inhibition of initiation observed by flow cytometry was not due to decreased amounts of mutant DiaA protein. Cell size was not significantly affected by expression of the mutant $\operatorname{dia} A$ alleles (data not shown).

Homo-oligomer formation of DiaA and the role of DiaA Asn83 and Lys101

Wild-type and mutant hisDiaA proteins were purified using Ni-NTA affinity column chromatography and then further purified using a gel-filtration column (Fig. 3A). The purity of the final protein fraction was $>90 \%$ for all DiaA proteins, based on SDS-PAGE analysis and Coomassie Brilliant Blue (CBB) staining (Fig. 3B).

The calculated molecular weight of a DiaA monomer is $\sim 23 \mathrm{kDa}$. Wild-type hisDiaA eluted from the gel-filtration column at a position corresponding to $50-60 \mathrm{kDa}$ (Fig. 3A), which indicated that DiaA forms homo-oligomers. Similar results were observed for the DiaA mutant proteins, with the exception of DiaA N83D and DiaA K101E. These two proteins eluted at a position corre- sponding to $40-50 \mathrm{kDa}$, which is consistent with hisDiaA homodimers (Fig. 3A), which indicated that these two mutant proteins fail to form homo-oligomers.

We further performed glycerol gradient ultracentrifugation experiments using wild-type hisDiaA, DiaA N83D, and DiaA K101E (Fig. 3C). Results were essentially consistent with those of gel filtration experiments. The sedimentation rate of wild-type hisDiaA corresponded to that of $100-60 \mathrm{kDa}$, which suggests that DiaA can form homotetramers. In contrast, sedimentation rates of DiaA N83D and DiaA K101E corresponded to that of $30-50 \mathrm{kDa}$.

These results correlated with the crystal structure data, which showed that Asn83 and Lys101 are located at the interface of a pair of homodimers. Together, the data of gel filtration, glycerol gradient ultracentrifugation, and crystal structure indicated that DiaA N83D and K101E form stable homodimers in solution. Wild-type DiaA most likely forms compactly folded homotetramers or equilibrates rapidly between homodimeric and homotetrameric states in the solution used for gel filtration. 
Figure 2. Timely initiation of replication is inhibited by diaA mutants. NA141 cells (diaA26::Tn5) carrying pKP1673 (vector) or pKP1673 derivatives encoding the indicated diaA alleles were grown exponentially at $30^{\circ} \mathrm{C}$ in LB medium containing thymine $(50 \mu \mathrm{g} / \mathrm{mL})$ and chloramphenicol $(10 \mu \mathrm{g} / \mathrm{mL})$ in the $(A)$ presence or (B) absence of $0.2 \%$ glucose to an $A_{660}$ of 0.2 . Portions of each culture were incubated for an additional $4 \mathrm{~h}$ in the presence of rifampicin and cephalexin. DNA content was then quantified using flow cytometry, and the equivalent chromosome number is shown. $(C, D) \mathrm{A}$ portion $(500 \mu \mathrm{L})$ of each culture was analyzed by immunoblot. NA141 cells (diaA26::Tn5) were used as the control for background immunoreactivity, and purified DiaA was added to the control whole-cell extract as a quantitative standard (Ishida et al. 2004). Bands that correspond to DiaA are indicated by arrows, and numbers under each panel indicate the amount of DiaA (in nanograms), as determined by the relative densitometric intensities.

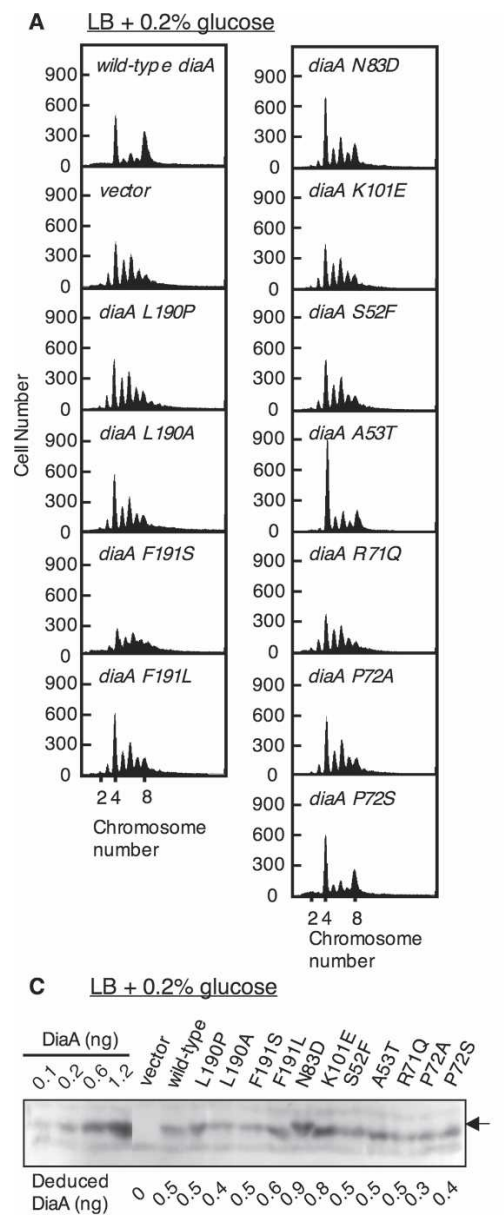

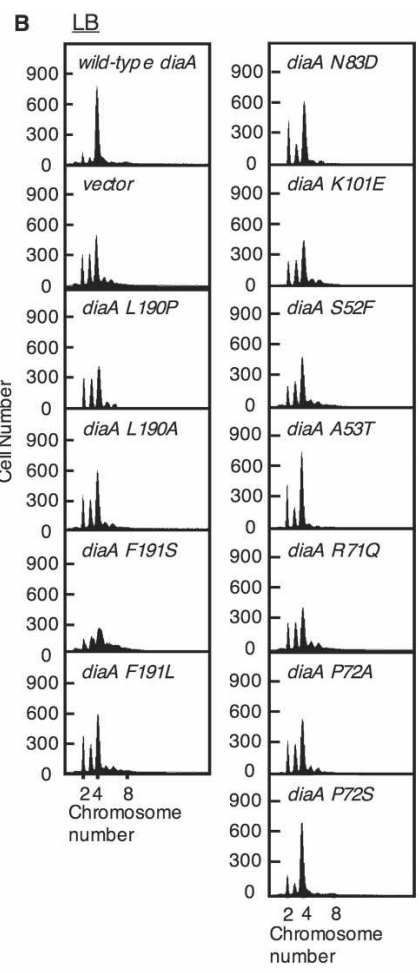

D $\underline{\underline{L B}}$

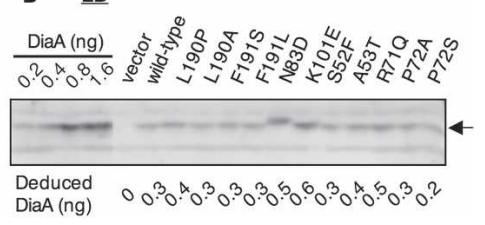

In addition, to determine if the disulfide bonds are required for DiaA oligomerization (Fig. 1C), we purified and analyzed DiaA C8S and C181S proteins. Results of gel filtration experiments indicated that DiaA C181S protein formed homo-oligomers like wild-type DiaA (Fig. 3A). DiaA C8S also showed similar results (data not shown). These results coincide with those of the in vivo complementation test as mentioned above.

\section{The mutant DiaA proteins fail to stimulate} minichromosomal replication in vitro

Minichromosomal replication in vitro is stimulated twoto threefold by DiaA (Ishida et al. 2004). The specific activity of wild-type hisDiaA was similar to that which was previously observed for nontagged DiaA (data not shown; Ishida et al. 2004). We then examined whether mutant DiaA proteins retained the ability to stimulate minichromosomal replication (Fig. 4). Under conditions in which the level of DnaA was limiting, in contrast to wild-type DiaA, none of the DiaA mutants stimulated minichromosomal replication (Fig. 4A-D). In the presence of increasing amounts of DnaA, wild-type DiaA stimulated replication approximately twofold (Fig. 4E), while the DiaA mutants had no stimulatory or inhibitory effect (Fig. 4E-G). These results indicated that the mutant dia $A$ derivatives were defective in the stimula- tion of DnaA-dependent initiation of replication in vitro, which was consistent with their activity in vivo (Table 1; Fig. 2). Since DiaA N83D and DiaA K101E most likely exist as stable homodimers (Fig. 3A), these results are also consistent with the idea that DiaA homodimers are inactive and that the formation of tetrameric DiaA is crucial for the stimulation of replication initiation.

When the representative mutant DiaA proteins (S52F, $\mathrm{N} 83 \mathrm{D}$, and L190A; 2 pmol) were incubated in replication reactions containing 1 pmol of DnaA and various amounts (0.25-2.0 pmol) of wild-type DiaA, the stimulation of replication was not affected (data not shown). Also, mixtures (1:1) of the two different mutant DiaA proteins were inactive in the stimulation of replication in vitro (data not shown).

Whereas DiaA P72S was inactive in the initiation stimulation in vitro (Fig. 4A), initiation of the diaA P72S cells was not inhibited in vivo in the presence of the wild-type dnaA gene (Fig. 2). DiaA P72S protein might be efficiently stimulated by chaperon proteins in vivo, sustaining interaction with wild-type DnaA, but not DnaAcos (Table 1).

\section{DiaA L190 and F191 mutants fail to bind DnaA}

It was shown previously using a pull-down assay and a biotin-tagged form of DnaA (bioDnaA) that DiaA binds 
A

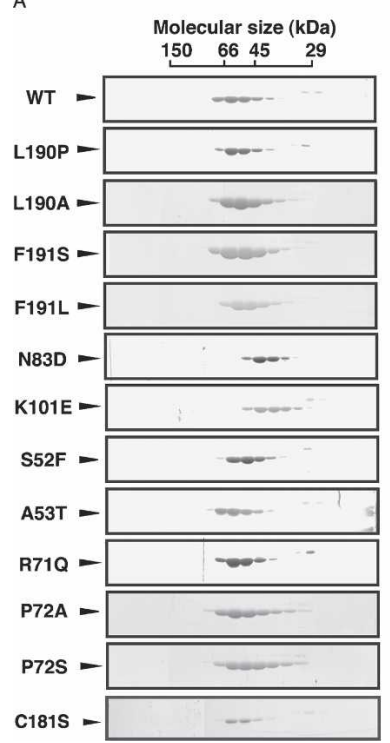

B
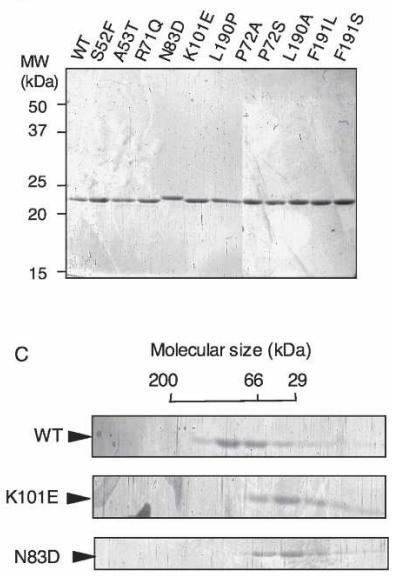

Figure 3. Oligomer formation analysis of wild-type and mutant DiaA proteins. (A) Wild-type hisDiaA (WT) and DiaA mutants (S52F, A53T, R71Q, P72A, P72S, N83D, K101E, L190P, L190A, F191S, F191L, and C181S) were subjected to Superdex 75 HR 10/30 gel filtration chromatography (24-mL bed volume), and fractions $(5 \mu \mathrm{L})$ were analyzed by $13 \%$ SDS-PAGE and CBB staining. Molecular size markers were subjected to the same conditions as above, and their positions are indicated at the top of the gel. (B) Pooled fractions (500 ng of protein) of wild-type hisDiaA or the indicated DiaA mutants were analyzed by $13 \%$ SDS-PAGE and CBB staining. (MW) Molecular size markers. $(C)$ Wild-type hisDiaA (WT), N83D, and K101E mutant proteins (20 pmol as monomer) were analyzed using a glycerol gradient $(15 \%-35 \%)$ ultracentrifugation method. After centrifugation, 11 fractions $(200 \mu \mathrm{L})$ were collected from the bottom of the tube and analyzed by $13 \%$ SDS-PAGE and CBB staining. Sedimentation positions of size marker proteins are indicated.

directly to DnaA and that the affinity of DiaA for DnaA was independent of the nucleotide-bound state of DnaA (Ishida et al. 2004). We performed a pull-down assay using bioDnaA and streptavidin-coated beads to determine whether mutant DiaA proteins retained the ability to bind to DnaA (Fig. 5A,B; Supplemental Fig. S3). The results indicated that DiaA L190A, L190P, F191L, and F191S were severely impaired in their ability to associate with DnaA, unlike wild type and the other mutant forms of DiaA. These results indicated that Leu190 and Phe191 of DiaA play a crucial role in its association with DnaA. Taken together, the results demonstrated that direct and specific binding between DiaA and DnaA is crucial for the stimulation of initiation.

The affinity of DiaA P72A and P72S for DnaA was severely decreased compared to wild type, although there was a small level of residual binding when DiaA was present in excess (Fig. 5A,B). This result was consistent with the tertiary structure data, in which Pro72 of DiaA is located close to Leu190 and Phe191 (Fig. 1). The other mutant DiaA proteins exhibited substantial DnaAbinding activity, although the level of binding was ap-

proximately half that of wild-type DiaA, even when DiaA was limiting (Fig. 5B), which indicated that the substitutions have an indirect effect on the interaction of DnaA and DiaA. DiaA N83D and K101E, which fail to form tetramers, were moderately impaired in binding to DnaA, which is consistent with the idea that DiaA tetramer formation is required for optimum binding of DnaA. We do not exclude a possibility that the moderate decrease in affinity for DnaA is an indirect consequence of a conformational change by the mutations. Moderately impaired activity of DiaA S52F and A53T in DnaA binding is consistent with the possibility that the structure of the phosphate-binding region is indirectly related to supporting of DnaA-binding activity. Like the wild-
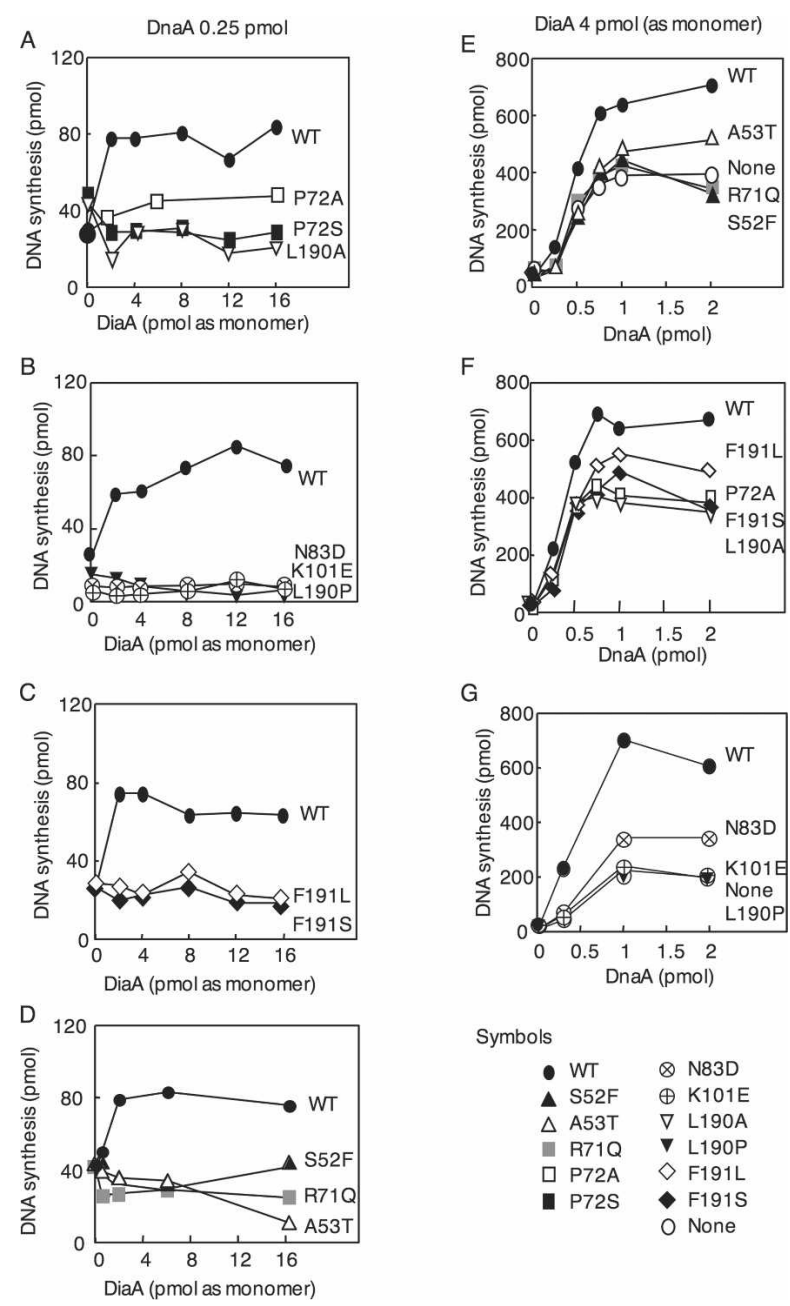

Figure 4. Analysis of DiaA mutants in DnaA-dependent replication of minichromosome in vitro. A crude replication extract was prepared from TK24 cells (dnaA204 diaA26:: Tn5) and used to assay replication of the oriC plasmid M13KEW101 (200 ng; 600 pmol nucleotide) in the presence of the indicated amounts of DnaA and hisDiaA. $(A-D)$ Reactions were incubated for 20 min at $30^{\circ} \mathrm{C}$ in the presence of DnaA $(0.25 \mathrm{pmol})$ and the indicated amounts of hisDiaA (WT) and DiaA mutants. $(E-G)$ The indicated amounts of DnaA were incubated as for $A-D$ in the presence of wild-type or the indicated mutants of hisDiaA /4 pmol monomer). 


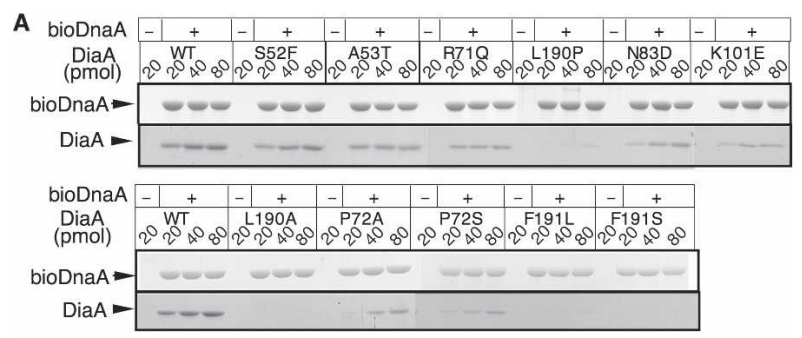

B
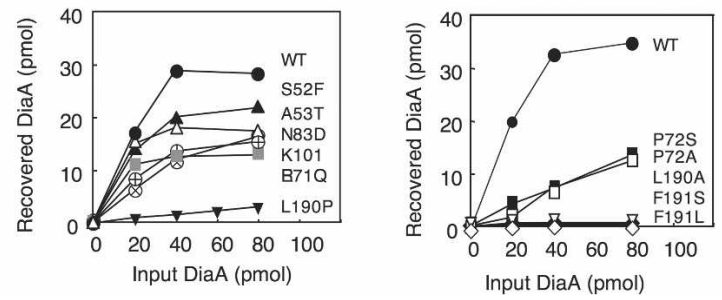

Figure 5. DiaA mutants in DnaA binding. $(A)$ Wild-type hisDiaA (WT) or DiaA mutants (S52F, A53T, R71Q, P72A, P72S, N83D, K101E, L190P, L190A, F191L, and F191S; 20-80 pmol monomer) were incubated for $5 \mathrm{~min}$ on ice in buffer $(50 \mu \mathrm{L})$ containing bioDnaA ( $40 \mathrm{pmol})$. Protein complexes were isolated using streptavidin beads, eluted in buffer containing 1\% SDS, and analyzed by $13 \%$ SDS-PAGE and CBB staining. $(B)$ The intensities of the bands in $A$ were quantified by densitometry, and the amounts of hisDiaA were determined using a standard curve (data not shown). The symbol key is shown in Figure 4.

type DiaA, these DiaA mutant proteins formed homomultimers (Fig. 3A), which indicated that overall denaturation of the mutant proteins was unlikely.

\section{Interaction of DiaA tetramers with multiple DnaA molecules}

To determine if multiple DnaA molecules bind to a single DiaA tetramer, we performed a two-stage pulldown assay. In the first stage of this experiment, we incubated $40 \mathrm{pmol}$ (as monomer) of DiaA molecules for 10 pmol of bioDnaA molecules. The monomer state of DnaA is stable in solution. Excessive amounts of DiaA should avoid that all of the DnaA-binding sites on DiaA tetramers are occupied with bioDnaA molecules only at the first stage. Protein complexes of bioDnaA and DiaA were isolated using streptavidin beads, then at the second stage the isolated complexes were incubated in buffer containing nontagged DnaA, and bound protein complexes were isolated and analyzed by SDS-PAGE. Recovery of nontagged DnaA increased in a manner depending on both DiaA and bioDnaA (Fig. 6A), which indicated that multiple DnaA molecules (bioDnaA and nontagged DnaA molecules) can bind to a single DiaA tetramer simultaneously. We observed similar results using ADP-DnaA (data not shown). The representative DiaA mutant proteins were similarly assessed (Fig. 6A). Recoveries of DiaA N83D and S52F proteins were decreased compared to those of wild-type DiaA. DiaA L190A was inactive in DnaA binding. These results are consistent with those shown in Figure 5, A and B.
Furthermore, we used another pull-down system in which hisDiaA was incubated in the presence of various amounts of DnaA, followed by recovery using $\mathrm{Co}^{2+}$-conjugated beads and determination of the amounts of proteins recovered (Fig. 6B). Results indicated that a single DiaA tetramer bound 1.6-2.3 DnaA molecules if all the DiaA tetramers were complexed with DnaA. If a portion of recovered DiaA tetramers were not associated with DnaA under these conditions, the number of DnaA molecules per DiaA tetramer would be elevated. Theses results are consistent with those of Figure 6A.

\section{DiaA enhances assembly of DnaA on oriC}

Based on the above results, we were interested in whether DiaA enhanced the assembly of DnaA molecules on oriC. DiaA binds to DnaA that is bound to the

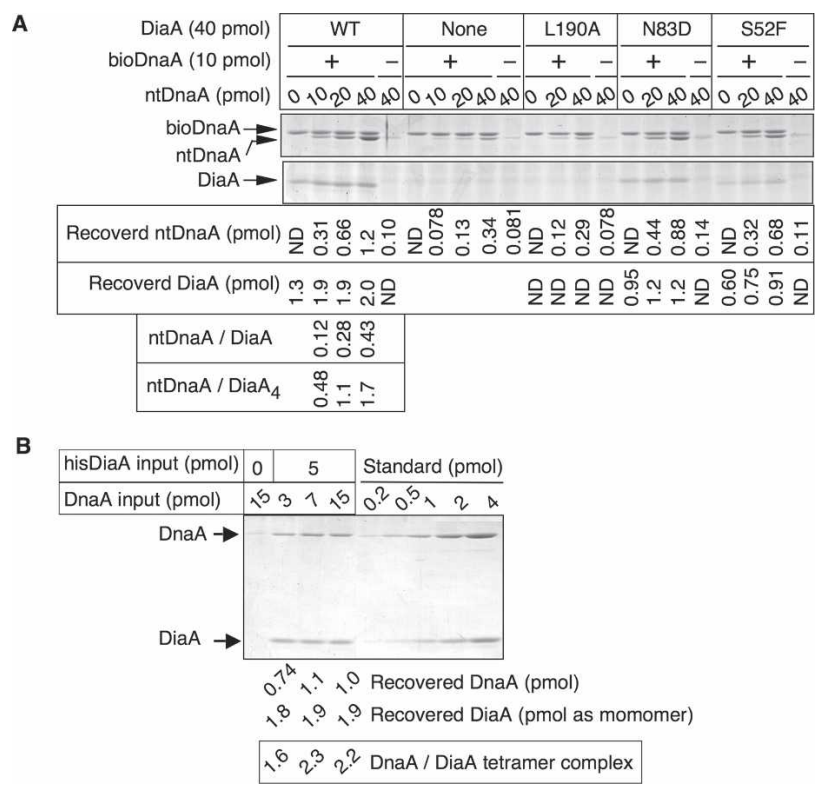

Figure 6. Binding modes of DiaA and DnaA. (A) Wild-type hisDiaA or DiaA mutants (S52F, N83D, and L190A; 40 pmol as monomer) were incubated for $5 \mathrm{~min}$ in the presence $(+)$ or absence (-) of bioDnaA (10 pmol). bioDnaA-hisDiaA complexes were isolated using streptavidin beads, and then incubated for 30 min at $4^{\circ} \mathrm{C}$ in buffer containing the indicated amounts of non-tagged DnaA (ntDnaA). Complexes were isolated and analyzed by $13 \%$ SDS-PAGE and CBB staining. The amounts of hisDiaA and ntDnaA were determined using a standard curve (data not shown) and are indicated below the gel image. The number of recovered ntDnaA molecules per DiaA or per DiaA tetramer was deduced (ntDnaA/DiaA and ntDnaA/DiaA 4 ). For calculation of this number, the slight nonspecific recovery of ntDnaA in the absence of DiaA was subtracted. Arrows indicate the positions of the proteins used in the assay. (ND) Not deleted. (B) Wild-type hisDiaA (5 pmol as monomer) was incubated for $15 \mathrm{~min}$ on ice in buffer containing indicated amounts of DnaA, followed by isolation using Dynabeads TALON (Invitrogen). Isolated proteins were washed, eluted in $60 \mathrm{mM}$ imidazole, and analyzed by $13 \%$ SDS-PAGE and CBB staining. The amounts of hisDiaA and ntDnaA were determined using a standard curve and are indicated below the gel image. The number of recovered DnaA molecules per DiaA tetramer was deduced. 

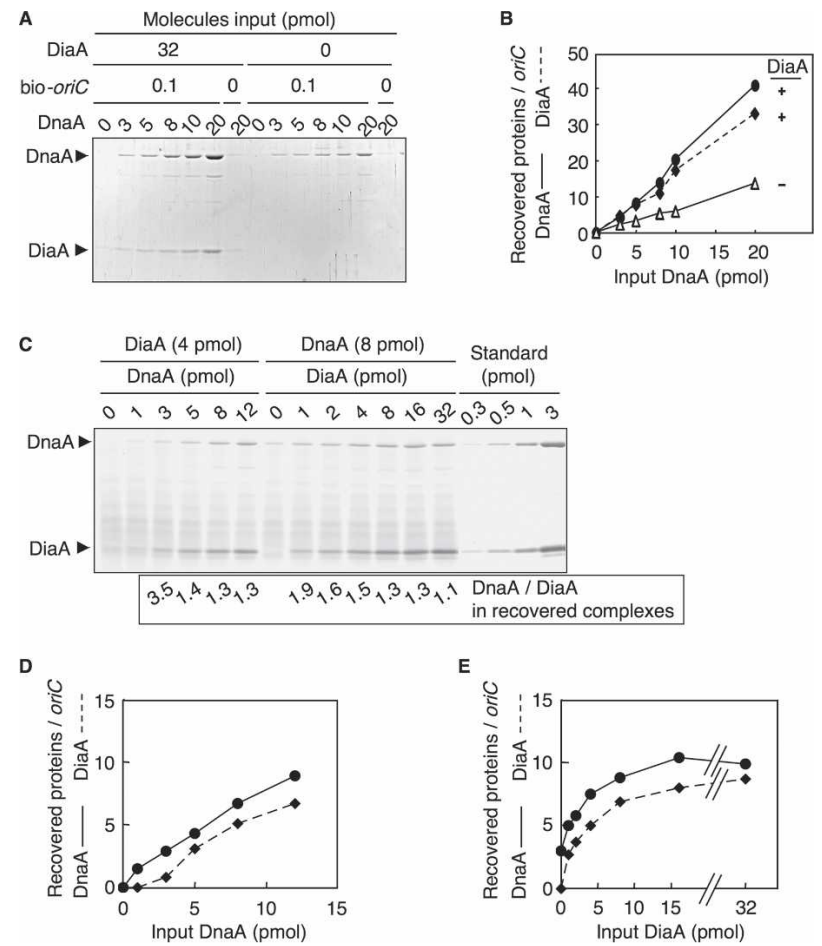

Figure 7. DiaA stimulates assembly of DnaA on oriC. $(A, C)$ The indicated amounts of DnaA were incubated for $30 \mathrm{~min}$ on ice in the presence or absence of hisDiaA and bio-oriC. Proteins bound to bio-oriC were isolated using streptavidin beads and analyzed by $13 \%$ SDS-PAGE and $(A)$ CBB staining or $(C)$ silver staining. $(B, D, E)$ The intensities of the bands were quantified by densitometry, and the amount of protein in complex with oriC was determined using a standard curve. $(C)$ As indicated in $A$, bio-oriC $(0.1 \mathrm{pmol})$ was also included in each reaction. The ratio of the number of DnaA molecules per DiaA monomer in each fraction is shown.

DnaA-box of oriC (Ishida et al. 2004). We incubated DnaA and DiaA with biotinylated-oriC DNA (bio-oriC, $419 \mathrm{bp}$ ) on ice, and DnaA-DiaA complexes that associated with oriC were isolated using streptavidin beads (Fig. 7). DiaA alone did not directly bind to oriC, but DnaA was recovered in an oriC-dependent manner, and the amount of DnaA bound to oriC increased proportionally with amount of input DnaA (Fig. 7A,B). In the presence of DiaA, the amount of DnaA bound to oriC was clearly enhanced in a dose-dependent manner (Fig. 7A-D).

When the amount of input DnaA was fixed at a limiting level, the amount of DnaA bound to oriC increased up to threefold in the presence of DiaA (Fig. 7C,E). This result was consistent with our previous results (Fig. 7AD), and with the stimulatory effect of DiaA on replication initiation (Fig. 4). Of note, this effect was observed when the ratio of DnaA to DiaA monomer was in the range of 2-4, which is similar to the ratio seen in vivo (Ishida et al. 2004). The recovered oriC-protein complexes contained DnaA and DiaA at an average ratio of 1.6:1 (Fig. 7C-E). Thus, if a single initiation complex is assumed to contain 20 DnaA molecules, it will contain only three DiaA tetramers. These results demonstrated that DiaA efficiently stimulates the assembly of DnaA molecules on oriC.

\section{DiaA mutants do not stimulate DnaA assembly on oriC}

We next examined whether three representative DiaA mutants-L190A, N83D, and S52F-stimulated the assembly of DnaA on oriC. DiaA L190A was specifically defective in DnaA-binding (Fig. 5). DiaA N83D retained considerable affinity for DnaA, but was defective in homotetramer formation (Fig. 3A). DiaA S52F retained both the modest DnaA-binding activity and the ability to form homotetramers, but was inactive in stimulating replication initiation in vivo and in vitro. As seen in Figure $8, \mathrm{~A}$ and $\mathrm{B}$, all the mutant proteins tested were severely impaired in their ability to stimulate the formation of DnaA-oriC complexes. The slight stimulatory activity of DiaA N83D and S52F could be due to DnaAbinding activity of the DiaA protomer. These results are consistent with the idea that full stimulatory activity of DiaA depends on both DnaA-binding activity and the

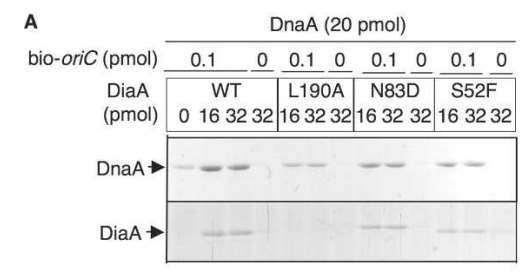

c

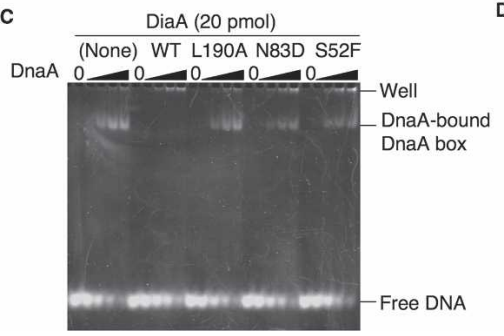

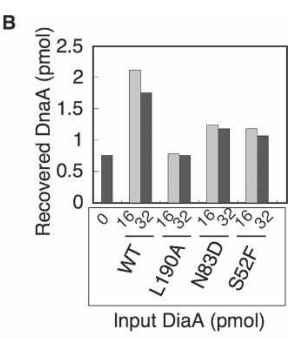

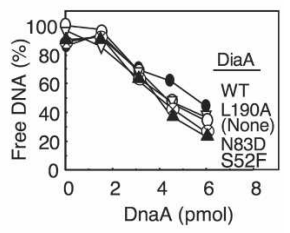

Figure 8. DiaA mutants are defective in stimulating the assembly of DnaA on oriC. (A) Stimulation of the assembly of DnaA on oriC was assessed as described for Figure 7. The indicated amounts (as monomers) of wild-type hisDiaA (WT) and DiaA mutants (L190A, N83D, and S52F) were incubated for 30 min on ice in buffer containing DnaA (20 pmol) and bio-oriC $(0.1 \mathrm{pmol})$. (B) The amounts of DnaA complexed with oriC were determined as described for Figure 7. $(C)$ The DnaA-box-binding activity of DnaA was assessed by gel-mobility retardation analysis. Various amounts of DnaA $(0,1.5,3,4.5$, and 6 pmol) were incubated for $20 \mathrm{~min}$ on ice in buffer containing a 15-mer DNA fragment (2.5 pmol) containing DnaA-box R1, in the presence or (None) absence of (WT) wild-type hisDiaA or DiaA mutants (L190A, N83D, and S52F; 20 pmol each). The samples were analyzed at $4^{\circ} \mathrm{C}$ by $8 \%$ PAGE and Gel-star staining. (D) The protein-free DNA (Free DNA) shown in $C$ was quantified, and the relative amounts in each sample were normalized to the level observed in the absence of both DnaA and DiaA [Free DNA (\%)]. The symbol key is shown in Figure 4. 
ability to form homotetramers. The requirement for Ser52 is consistent with the idea that the putative phosphosugar-binding surface is also an important functional structure. We performed gel-mobility shift experiments to confirm that wild-type DiaA and the three DiaA mutants did not inhibit the affinity of DnaA for the DnaAbox sequence (Fig. 8C,D). Efficiency in decrease of protein-free DnaA-box molecules was not affected by wildtype and mutant DiaA proteins. DiaA did not inhibit or stimulate the initial binding of DnaA to a single DnaAbox. A discrete band corresponding to a DiaA-DnaADnaA-box complex was not seen for wild-type DiaA. Most likely wild-type DiaA efficiently promoted oligomerization of the nucleoprotein complexes, resulting in assemblies that have higher molecular mass and cannot migrate into the gel.

\section{DiaA stimulates open complex formation}

We next performed a P1 nuclease assay to investigate whether DiaA promoted the formation of the oriC open complex (Kawakami et al. 2005, 2006). P1 nuclease specifically degrades single-stranded DNA. When plasmid ori $C$ is digested by $\mathrm{P} 1$ nuclease, subsequent digestion with the AlwNI restriction enzyme produces a $3.8-\mathrm{kb}$ and a 4.1-kb fragment (Fig. 9). In the presence of limiting amounts of DnaA, DiaA appeared to promote formation of the open oriC complex. Open complex formation was not stimulated by DiaA L190A, N83D, and S52F. These results were consistent with earlier results showing that DiaA stimulated the assembly of DnaA on oriC (Fig. 8).

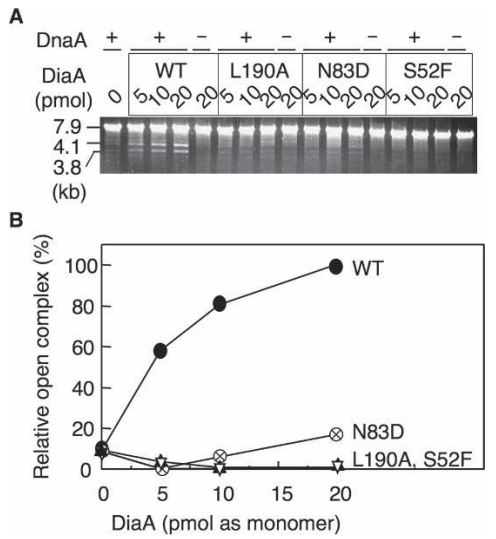

Figure 9. The wild-type DiaA, but not DiaA mutants, promotes oriC open complex formation. $(A)$ The indicated amounts (as monomers) of wild-type hisDiaA (WT) and DiaA mutants (L190A, N83D, and S52F) were incubated for $3 \mathrm{~min}$ at $38^{\circ} \mathrm{C}$ in buffer containing the oriC plasmid M13KEW101 (320 ng) and HU protein (13 ng), in the presence $(+)$ or absence $(-)$ of ATPDnaA (1.2 pmol), then incubated further in the presence of P1 nuclease. After digestion with AlwNI, the DNA fragments were analyzed by $1 \%$ agarose gel electrophoresis and ethidium bromide staining. (B) The intensities of the $3.8-\mathrm{kb}$ and $4.1-\mathrm{kb}$ fragments were quantified for each sample by densitometry, and the relative amount of each fragment is presented. The symbol key is shown in Figure 4.
Severe defects of DiaA S52F and N83D proteins in oriC unwinding is consistent with those of these proteins in the stimulation of DnaA assembly on oriC (Fig. 8A,B). Most likely these mutant proteins were impaired to stimulate the stable assembly of multiple DnaA molecules on oriC, and functional initiation complexes were not formed (see below). Stimulation of open complex formation required higher levels of DiaA per molecule of DnaA than the stimulation of minichromosome replication (Fig. 4) and DnaA assembly on oriC (Fig. 8). The minichromosome replication reaction contained crude protein extracts, which might include a factor that stimulates the activity of DiaA in promoting a conformational change of assembled DnaA multimers on oriC, and open complex formation. ADP-DnaA did not stimulate open complex formation even in the presence of DiaA (data not shown).

\section{DiaA stimulates activated state of initiation complex}

To determine whether DiaA promoted the formation of higher-order ATP-DnaA-oriC complexes, we performed a DNase I footprinting analysis of oriC (Kawakami et al. 2005). The oriC region carries high-affinity DnaA-boxes and low-affinity sites that ATP-DnaA specifically binds (Margulies and Kaguni 1996; McGarry et al. 2004; Kawakami et al. 2005). Initiation depends on the interaction of ATP-DnaA with the specific low-affinity sites (McGarry et al. 2004; Kawakami et al. 2005). Our previous study had shown that DnaA specifically binds with high affinity to DnaA-box sequences (termed R1 and R4) at a concentration of $100 \mathrm{nM}$ DnaA, in a manner independent of the nucleotide-bound state of DnaA (Kawakami et al. 2005). At a concentration of $500 \mathrm{nM}$, DnaA interacts with several additional unique sites (termed I-sites, $\tau$-sites, M, R2, and R3) in an ATP-dependent manner (Kawakami et al. 2005). DiaA promoted the association of ATP-DnaA with each of these specific binding sites, even at a DnaA concentration of $100 \mathrm{nM}$ (Fig. 10). In the absence of DnaA, DiaA alone did not appear to bind to oriC.

The DiaA L190A, N83D, and S52F mutants were unable to stimulate formation of ATP-DnaA complexes on oriC, although DiaA N83D showed slight residual activity (Fig. 10). In the presence of ADP-DnaA, DiaA stimulated the association of DnaA molecules specifically with a region between DnaA-boxes $\mathrm{R} 2$ and $\mathrm{R} 4$, but the digestion pattern was different from the ATP-DnaA footprint. These results were consistent with the P1 nuclease assay and indicated that DiaA functions to stimulate the formation of active initiation complexes by promoting the interaction of ATP-DnaA with its specific binding sites.

\section{DiaA-dependent stimulations of initiation in vivo}

Disruption of the diaA gene exhibits unstable maintenance of minichromosomes and synthetic lethality with specific dnaA mutant alleles at $37^{\circ} \mathrm{C}$ (Ishida et al. 2004). 


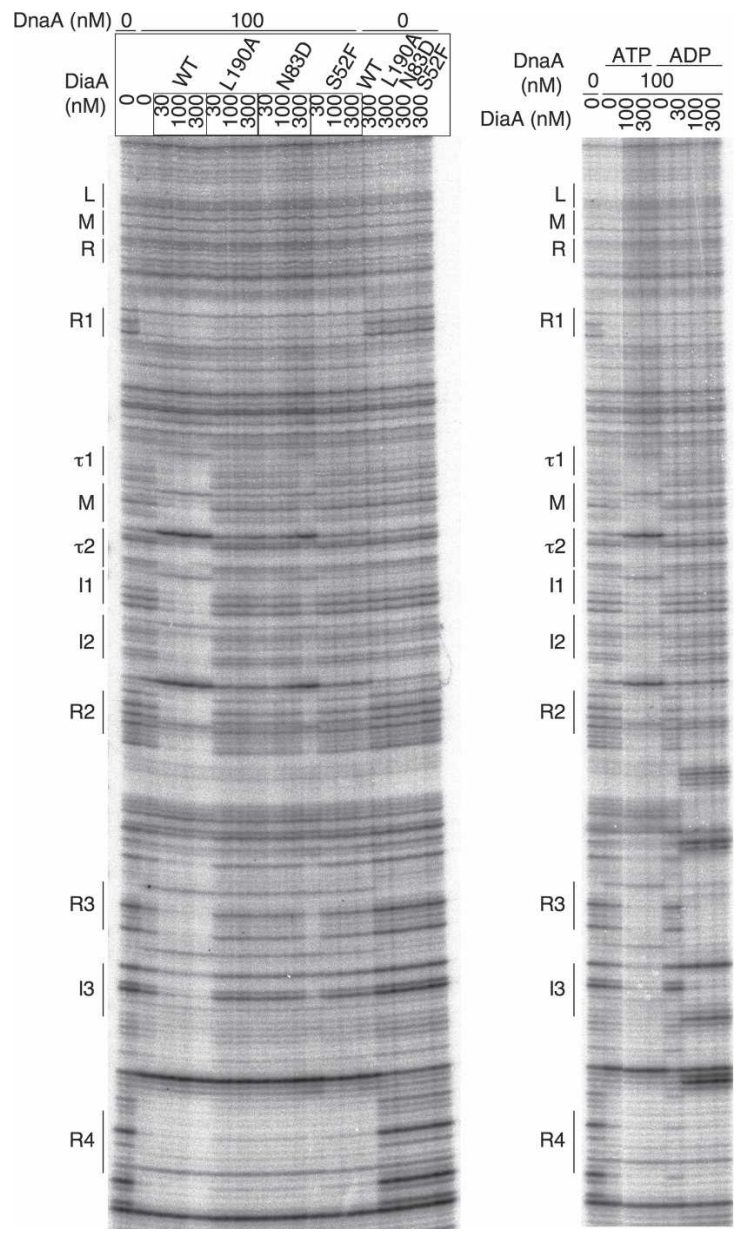

Figure 10. DiaA promotes ATP-DnaA-specific oriC complex formation. The indicated amounts (as monomers) of wild-type hisDiaA (WT) and DiaA mutants (L190A, N83D, and S52F) were incubated for $10 \mathrm{~min}$ at $30^{\circ} \mathrm{C}$ in buffer containing a ${ }^{32} \mathrm{P}$-endlabeled oriC fragment (419 bp) and $3 \mathrm{mM} \mathrm{ATP}$, in the presence or absence of DnaA, then incubated for $4 \mathrm{~min}$ at the same temperature in the presence of DNase I. When ADP-DnaA was assessed (right panel), the reaction buffer included $3 \mathrm{mM}$ ADP instead of ATP. The resultant DNA fragments were analyzed by $5 \%$ sequencing gel electrophoresis. The positions of DnaA-binding sequences (boxes R1-R4 and $\mathrm{M}$; sites I1-I3 and $\tau 1-\tau 2$ ) and the 13-mer AT-rich repeats (L, M, and R) are indicated.

To further analyze the in vivo role for DiaA in stimulation of initiation complex formation, we assessed the sensitivity of a diaA-null strain (NA141) to elevated levels of ATP-DnaA. DnaA R334A has an alanine substitution for the crucial arginine residue within the AAA+ sensor II motif. Our previous study reveals that ATPDnaA R334A mutant protein is defective in hydrolysis of the bound ATP, whereas this protein is associated with intact activities in ATP/ADP binding and initiation at oriC (Nishida et al. 2002). The ATP-form DnaA R334A molecules form active initiation complexes like the ATP-form wild-type DnaA molecules. As ATP bound to DnaA R334A is not hydrolyzed by RIDA, the level of the ATP-form DnaA molecules is increased in cells bearing this mutant DnaA, resulting in severe overinitiation at ori $C$ and inhibition of colony formation of cells (Nishida et al. 2002). Plasmids pSN306 and pSN305 are derivatives of pBR322 bearing a wild-type dnaA- or dnaA $R 334 A$-coding region, respectively, that is located downstream from the lac promoter. IPTG (isopropyl- $\beta$-D-thiogalactopyranoside) induces moderate increase of DnaA molecules in the cells. In the presence of IPTG, growth of the wild-type diaA cells was severely inhibited in a DnaA R334A-dependent manner, whereas that of diaAnull cells was not (Fig. 11A). These results are consistent with the idea that DiaA plays an important role in stimulation of initiation complex formation by ATPDnaA molecules.

Cell cycle parameters of a diaA mutant strain (NA141) containing pBR322 or a dnaA-carrying pBR322-derivative (pHB10S) were also analyzed using flow cytometry. Results indicated that the number of origins per cell mass was increased in a pHB10S-dependent manner (data not shown), which suggests that overexpression of DnaA can suppress the diaA mutation at least in the inhibition

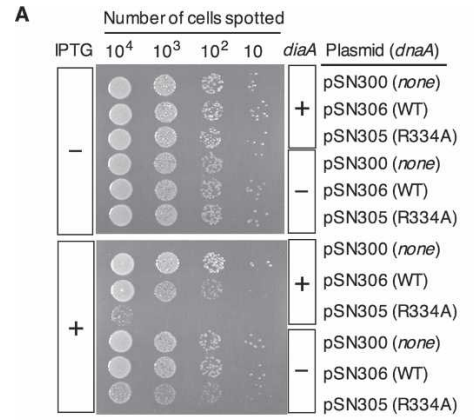

B

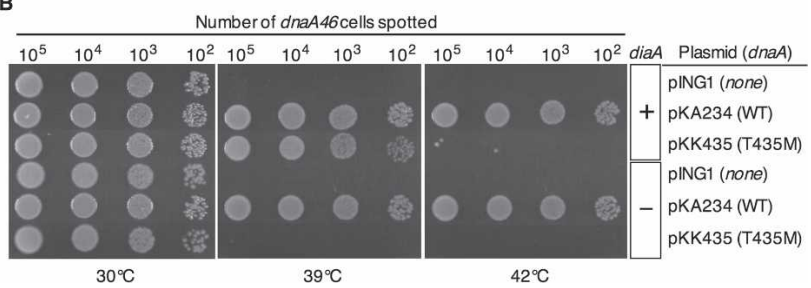

Figure 11. DiaA-dependent activation of DnaA initiation function in vivo. (A) Cells of KH5402-1 (wild-type diaA) and NA141 (diaA : $\operatorname{Tn} 5$ ) carried pHSL99 bearing the lac $^{\mathrm{q}}$ gene and pSN300derivative plasmid. $\left(\mathrm{dia}^{+}\right)$KH5402-1 (pHSL99) or $\left(\mathrm{diaA}^{-}\right)$ NA141 (pHSL99) cells carrying pSN300 [vector (none)], pSN306 [wild-type dnaA (WT)], and pSN305 [dnaA R334A (R334A)] were grown overnight at $37^{\circ} \mathrm{C}$ in LB medium containing thymine $(50 \mu \mathrm{g} / \mathrm{mL})$, ampicillin $(50 \mu \mathrm{g} / \mathrm{mL})$, and tetracycline $(7.5$ $\mu \mathrm{g} / \mathrm{mL}$ ). Three-microliter serious dilutions of the resultant cells (the $A_{660}$ of $3.0 ; \sim 10^{9}$ cell $/ \mathrm{mL}$ ) were spotted on LB agar plates containing the above chemicals in the presence $(+)$ or absence of IPTG $(0.1 \mathrm{mM})$ and incubated for $20 \mathrm{~h}$ at $30^{\circ} \mathrm{C}$. (B) Cells of $\left(d i a A^{+}\right)$KA413 (dnaA46) and $\left(\right.$dia $\left.^{-}\right)$KA467 (dnaA46 diaA::Tn5) bearing pING1, pKA234 (wild-type $d n a A$ ), or pKK435 (dnaA T435M) were grown overnight at $30^{\circ} \mathrm{C}$ in LB medium containing thymine $(50 \mu \mathrm{g} / \mathrm{mL})$ and ampicillin $(50 \mu \mathrm{g} /$ $\mathrm{mL}$ ). Three-microliter serious dilutions of the resultant cells were spotted on LB agar plates containing the above chemicals and incubated for $24 \mathrm{~h}$ at $30^{\circ} \mathrm{C}$ or for $15 \mathrm{~h}$ at $39^{\circ} \mathrm{C}$ or $42^{\circ} \mathrm{C}$. 
of initiation. This is consistent with the idea described above. Initiations of multiple origins in a single cell were asynchronous in DnaA-overexpressing cells regardless of the presence or absence of the diaA gene.

The dnaA46 mutant allele produces a mutant DnaA protein bearing substitution mutations within domain III and colony formation of the dnaA46 cells is temperature $\left(39^{\circ} \mathrm{C}-42^{\circ} \mathrm{C}\right)$ sensitive (Erzberger et al. 2002; Ishida et al. 2004). DnaA C-terminal domain IV has a helixturn-helix DNA-binding motif that is required for DnaA-box binding (Bleasing et al. 2000; Fujikawa et al. 2003). DnaA T435M protein bears a substitution within domain IV and is inactive in initiation due to a specific defect in DnaA-box binding (Sutton and Kaguni 1997; Bleasing et al. 2000; Fujikawa et al. 2003). We introduced plasmids bearing a wild-type dnaA or dnaA T435M allele (pKA234 or pKK435, respectively) in a dnaA46 mutant (KA413) and in a dnaA46 diaA-null mutant (KA467) at $30^{\circ} \mathrm{C}$ (Fig. 11B). When KA413 cells bearing pKA234 or pKK 435 were incubated at $39^{\circ} \mathrm{C}$ but not $42^{\circ} \mathrm{C}$, colonies were formed at a similar efficiency. This suppression might occur by formation of mixed complexes of DnaA46 and DnaA T435M proteins on oriC, resulting in initiation (Kawakami et al. 2005). DnaA46 is intact in the DNA-binding domain, and DnaA T435M is intact in domain III, which carries the DnaA-DnaA interaction activity. Alternatively, the residual activity of DnaA $\mathrm{T} 435 \mathrm{M}$ might be somehow elevated at $39^{\circ} \mathrm{C}$. This suppression of the dnaA46 cells bearing pKK435 was dependent on wild-type diaA (Fig. 11B). These results are also consistent with idea that DiaA can stimulate DnaA assembly on oriC.

\section{Homology analysis of DiaA}

We searched for E. coli DiaA homologs in a genome database. The DiaA C terminus bears Leu190 and Phe191 residues that are crucial in DnaA binding, whereas $E$. coli GmhA does not conserve these residues at the corresponding position (Figs. 1A and 5A). We used this feature to possibly distinguish between DiaA and GmhA homologs. DiaA homologs were found in various species in the eubacterial domain (Table 3). Especially many species in the $\gamma$-Proteobacterial division, to which E. coli belongs, conserve DiaA homologs. Of note, a considerable number of species bears a DiaA homolog but does not a GmhA homolog. Only for the $\beta$-Proteobacterial division, instead of the Leu-Phe residues, Leu-Leu residues are conserved of the homologs at the corresponding position. We defined these as DiaA homologs, based on similarity of the amino acid sequence and conservation of the putative operon structure. Like the E. coli diaA gene, the ORFs of these homologs are located between the ORFs of yraN and yraP on the genome.

\section{Discussion}

DiaA is a DnaA-binding protein that stimulates the initiation of chromosomal replication and is required to
Table 3. Conservation of DiaA homologs among eubacterial species

\begin{tabular}{|c|c|c|c|}
\hline \multirow[b]{2}{*}{ Phylum } & \multirow[b]{2}{*}{ Class } & \multicolumn{2}{|c|}{$\begin{array}{c}\text { Number of } \\
\text { species carrying }\end{array}$} \\
\hline & & $\begin{array}{c}\text { DiaA homolog } \\
\text { and GmhA } \\
\text { homolog }\end{array}$ & $\begin{array}{c}\text { DiaA } \\
\text { homolog } \\
\text { only }\end{array}$ \\
\hline \multirow[t]{4}{*}{ Proteobacteria } & $\gamma$-Proteobacteria & 53 & 41 \\
\hline & $\beta$-Proteobacteria & 5 & 27 \\
\hline & $\delta$-Proteobacteria & 1 & 14 \\
\hline & $\varepsilon$-Proteobacteria & 10 & 6 \\
\hline Chloroflexi & Chloroflexi & 2 & 2 \\
\hline Spirochaetes & Spirochaetes & 3 & 0 \\
\hline Planctomycetes & Planctomycetacia & 0 & 2 \\
\hline Lentisphaerae & & 0 & 1 \\
\hline Firmicutes & Clostridia & 0 & 1 \\
\hline Actinobacteria & Actinobacteria & 3 & 0 \\
\hline Total & & 77 & 94 \\
\hline
\end{tabular}

Based on the amino acid sequences, E. coli DiaA and GmhA homologs were searched using the BLAST program and the NCBI nonredundant (NR) database. Of homologs that have $>40 \%$ similarity, proteins that conserve the sequence of LeuPhe (or Leu-Leu only in $\beta$ Proteobacteria) corresponding to Leu190 and Phe191 of E. coli DiaA are defined as DiaA homologs. Proteins that lack this conservation are defined as GmhA homologs. Genomes of 171 species are found to carry both the DiaA and GmhA homologs or only the DiaA homolog (see also Supplemental Table S2 in detail).

ensure timely initiation during the cell cycle in E. coli. In this study, we revealed the crystal structure of DiaA and carried out a structure-function analysis of DiaA to gain a better understanding of the mechanism of stimulation of initiation by DiaA. DiaA forms homotetramers through the symmetrical pairing of stable homodimers. Mutational analysis of DiaA indicated that the tetramer form of the protein is the active form for stimulating the initiation of replication, while the dimerized form is inactive. We defined a specific site in the $\mathrm{C}$ terminus of DiaA encompassing Leu190 and Phe191 that is required for binding DnaA. We showed that a single DiaA tetramer can bind multiple molecules of DnaA and that the direct interaction of DnaA and DiaA is crucial for the regulation of initiation by DiaA. Previously, we demonstrated that the DnaA N-terminal domains I-II bind to DiaA (Ishida et al. 2004). Domain III and the C-terminal domain IV of DnaA are associated with ATP/ADP-binding and DNA-binding activities, respectively (Erzberger et al. 2002; Messer 2002; Fujikawa et al. 2003; Kawakami et al. 2005, 2006; Kaguni 2006). DiaA stimulated the formation of DnaA multimers on oriC, which stimulated the formation of open complexes. Binding of ATP-DnaA to specific sites within oriC was stimulated by DiaA. Thus, we have defined the mechanism by which DiaA stimulates initiation at oriC.

The DiaA tetramer can bind multiple molecules of DnaA, and thus can serve as a bridge to bring two DnaA molecules together. Binding of DnaA to the DiaA tetramer may serve as a mechanism to overcome the ef- 
fects of random diffusion and facilitate inter-DnaA interactions. This linkage effect would also explain the stimulation of DnaA binding to DNA by DiaA. The linkage effect has been observed for several DNA-binding proteins (Zhou 2001; Stauffer and Chazin 2004). The two domains of RPA70 have modest single-stranded DNAbinding activity alone. When the domains are connected by a flexible linker, the overall affinity for singlestranded DNA is enhanced 1000-fold (Arunkumar et al. 2003). The $\lambda c I$ repressor has specific domains for DNA binding and dimerization, and dimerization is required for high-affinity binding to DNA (Burz et al. 1994). Similarly, our results suggest that a single DnaA molecule of the DiaA-DnaA complex binds strongly to a DnaA-box sequence, and then this event stimulates binding of a second DnaA molecule in the complex to a flanking DNA site, resulting in the formation of stable DnaA multimers on oriC. DiaA had no stimulatory activity for the initial binding of DnaA to a single DnaA-box (Fig. $8 \mathrm{C}, \mathrm{D})$, which is also consistent with the above idea.

During the cell cycle, the fluctuation of ATP-DnaA levels is coupled to replication initiation. In the dnaC2 (Ts) mutant, when the cell cycle is synchronized, the level of ATP-DnaA increases from a basal level and reaches a peak after $10 \mathrm{~min}$, which is the point at which replication initiates (Kurokawa et al. 1999). Thus, there is a limited time span in which ATP-DnaA molecules must assemble on oriC to ensure the initiation of replication in a timely and synchronized manner. The results of the present and previous studies indicate that DiaA plays a specific and crucial role in this process, and that when DiaA is absent or inactive, initiation is delayed, resulting in reduced oriC copy number and asynchronous initiations. In a cell that contains two copies of oriC, replication initiation at only one of the two sites due to improper regulation of preinitiation events results in activation of the RIDA system, and reduction of ATPDnaA levels. Thus, replication initiation at the second oriC is inhibited. Regulation of pre-initiation events to ensure that replication initiation occurs synchronously and at the proper time is profoundly important for proper maintenance of the replication cycle.

In addition, an idea that there might be cross-talk between the oriC copies in a single cell to ensure the synchrony of initiation would be possible. Unknown factors and mechanisms might be responsible for cross-talk of the oriC copies, which might be affected by the lack of DiaA. We searched for DiaA-binding proteins and found several candidates (data not shown). Functional analysis of these factors is in progress.

Although the DiaA S52F and A53T mutant proteins formed homotetramers and were able to bind to DnaA, they were defective in the stimulation of initiation, open complex formation, and formation of ATP-DnaA-specific oriC complexes. These residues are located in the putative phosphosugar-binding region of DiaA, but we cannot exclude the possibility that mutation of these residues disrupts internal interactions of DiaA that affect the overall structure of the protein. Another possibility is that these mutations, indeed, affect the binding of DiaA to phosphosugars and that this association regulates the activity of DiaA. If phosphosugar binding is essential to activity, mutants that fail to bind phosphosugars would be inactive. Alternatively, a mutation might result in a protein that binds phosphosugars more tightly than wild-type DiaA, which may result in constitutive inactivation. Phosphosugars such as inositoltriphosphate and inositol-hexakisphosphate are known to regulate protein function in sugar metabolic pathways and in signal transduction pathways (Hanson et al. 2004; Kim et al. 2006). The YrbH protein of pathogenic Yersinia pestis has an SIS domain and arabinose-5-phosphate isomerase activity, and is required for biofilm formation (Tan and Darby 2006). However, to date, phosphosugars have not been implicated in the regulation of replication initiation. At least, glucose did not affect the affinity of DiaA for DnaA in vitro (data not shown).

We do not exclude a possibility that DiaA function can be regulated during the cell cycle, although at present we do not have concrete evidence to prove the cell cyclecoordinated functional change of DiaA. As DiaA has a phosphosugar-binding motif, one of possible ideas is that a specific phosphosugar binds to DiaA in a cell cycledependent manner, which regulates DiaA function such as DnaA binding or tetramer formation.

The oriC region contains several DnaA-binding sites of varying affinity (Margulies and Kaguni 1996; Weigel et al. 1997). DnaA R1 and R4 boxes are associated with high affinity for DnaA, independent of the nucleotide-bound state of DnaA. Other DnaA-boxes are associated with lower affinity for DnaA, and some of them specifically interact with ATP-DnaA. ATP-DnaA binding to these weak-affinity sites is crucial for open complex formation (McGarry et al. 2004; Kawakami et al. 2005). We demonstrated that DiaA stimulated the interaction of ATPDnaA with ATP-DnaA-specific sites of oriC, which most likely promotes the formation of open complexes. In contrast, DiaA did not stimulate the binding of ADPDnaA molecules to ATP-DnaA-specific sites such as I1, I2, $\tau 1, \tau 2$, and $M$ sites (Fig. 10). This indicates that the integrity of ATP-DnaA-specific DNA binding is preserved in the presence of DiaA. The formation of ATPDnaA-specific oriC complexes depends on Arg285 of DnaA, which plays a key role in inter-DnaA interactions (Kawakami et al. 2005; Erzberger et al. 2006). One implication of our results is that DiaA reinforces this ATPDnaA-specific inter-DnaA interaction. This is consistent with the results of the pull-down experiments, the minichromosomal replication assay, and the oriC unwinding assay.

In the presence of DiaA and ADP-DnaA molecules, protection from DNase I was seen only for DnaA-boxes $\mathrm{R} 1$ and R4 and a region between DnaA-boxes R2 and R4 (Fig. 10). DnaA-boxes R1 and R4 have most potent affinity for DnaA regardless of the nucleotide forms. Unlike DnaA-box R1, box R4 is adjacent to the sites (I3, boxes R3 and R2) that DnaA can weakly bind. The I3 site has a sequence carrying a DnaA-box 9-mer consensus with three mismatches (McGarry et al. 2004; Kawakami et al. 2005). We speculate that DiaA would stimulate interac- 
tion of DnaA molecules to DnaA tightly complexed with box R4, resulting in binding of ADP-DnaA to these R4adjacent weak-affinity sites. Also, DiaA-dependent footprint patterns for this region (R4 to R2) are clearly different between ATP-DnaA and ADP-DnaA. This suggests differences in the mode of inter-DnaA interaction. Most likely this difference can be caused by the presence or absence of interaction between ATP and the DnaA arginine finger Arg285 (Kawakami et al. 2005).

DiaA forms homotetramers, which appear to be the active form of the protein that stimulates replication initiation. The results of gel filtration analysis showed that wild-type DiaA eluted at a position corresponding to a molecular mass of $50-60 \mathrm{kDa}$, which is smaller than the calculated molecular mass of a DiaA homotetramer. In a dilute solution of DiaA, there may be a state of equilibrium between homotetramers and homodimers. A previously purified preparation of non-tagged DiaA may contain predominantly homodimers (Ishida et al. 2004).

We previously indicated that DiaA binds to an N-terminal domain of DnaA (Ishida et al. 2004). Recently, we revealed the tertiary structure of the DnaA N-terminal domain and reported that the Glu21 residue, which is exposed on the protein surface of the domain, is specifically required for DnaB helicase loading (Abe et al. 2007). The Phe46 residue of this domain is also exposed on the protein surface. We find that DnaA F46A mutant protein is active in ATP binding but is defective in DiaA binding in vitro (data not shown).

\section{Materials and methods}

Strains and plasmids

E. coli K12 derivatives KH5402-1 (wild-type), NA001 (dnaAcos), NA26 (dnaAcos diaA26::Tn5), NA141 (diaA26::Tn5), TK24 (dnaA204 diaA26::Tn5), and KA413 (dnaA46) were previously described (Ishida et al. 2004; Kawakami et al. 2005). KA467 is a KA413 derivative carrying diaA26::Tn5. pBAD/HisB was purchased from Invitrogen. The oriC plasmid M13KEW101 was previously described (Kawakami et al. 2005). To construct the plasmid encoding hexahistidine-fused DiaA, a diaA fragment $(0.56 \mathrm{~kb})$ was amplified using pNA159 (Ishida et al. 2004) as the template by PCR, using the primers TAKU15 $\left(5^{\prime}\right.$-CCGGCTAG CATGCAAGAAAGAATTAAAGCT-3') and TAKU4 (5'-CGG AATTCCTCCTTAATCATCCTGGTG-3'). The amplified PCR product was cloned into pBAD/HisB using the NheI and EcoRI restriction sites to generate pTKM601. The single base substitutions of pdiaA P72A, pdiaA P72S, pdiaA L190A, pdiaA C8S, and pdiaA C181S were generated using the QuikChange sitedirected mutagenesis kit (Stratagene) and pTKM601 as the template (Supplemental Table S1). A 2.4-kb NdeI fragment from pMn21 and pMn54, containing diaA F191S and diaA F191L, respectively, was ligated to a $2.1-\mathrm{kb}$ NdeI fragment derived from pTKM601 to generate pdiaA F191S and pdiaA F191L. All mutations were confirmed by nucleotide sequencing.

A $0.56-\mathrm{kb}$ HindIII-EcoRI fragment bearing diaA with the Histag deleted was isolated from pTKM601 and inserted into the corresponding sites of the low-copy mini-R (pKP1673) derivative plasmid pNA102, to generate pTKM701. pNA102 encodes non-tagged wild-type diaA (Ishida et al. 2004). Derivatives of pNA102 encoding mutant diaA alleles were constructed in a similar manner (Table 1; see below).
Plasmids pSN300, pSN305, pSN306, and pHSL99 were previously described (Nishida et al. 2002). Briefly, pSN300 was a pBR322 derivative bearing a lac promoter, and pSN305 and pSN306 were pSN300 derivatives bearing a dnaA R334A and wild-type dnaA coding region, respectively, downstream from the lac promoter. pHSL99 is a pACYC184 derivative bearing the $1 a c I^{\mathrm{q}}$ gene. Plasmid pKK435 bearing dnaA T435M was constructed using pKA234, the site-directed mutagenesis kit as above, a primer 5'-CGTTTGGTGGCCGTGACCATATGACG GTGCTTCATGCCTGC-3', and its complementary primer. pKA234 was a derivative of pING1 that overproduces wild-type DnaA in an arabinose-inducible manner (Kawakami et al. 2005).

\section{Introduction of mutations and selection of dysfunctional} DiaA mutants

The mutagenic PCR method was used as previously described, with minor modifications (Guo et al. 1999). Briefly, diaA (0.56$\mathrm{kb}$ fragment) was amplified by PCR using pTKM601 as the template, and the primers TAKU15 and TAKU4, in the presence of $0.05 \mathrm{mM}$ or $0.1 \mathrm{mM}$ manganese. The amplified diaA-coding region was inserted in pBAD/HisB using the NheI and EcoRI restriction sites. To isolate dysfunctional DiaA mutants, the plasmids were introduced into NA26 cells, and transformed cells were obtained by incubation for $20 \mathrm{~h}$ at $30^{\circ} \mathrm{C}$ on LB agar plates containing $0.05 \% \mathrm{~L}(+)$-arabinose, $100 \mu \mathrm{g} / \mathrm{mL}$ ampicillin, and $25 \mu \mathrm{g} / \mathrm{mL}$ thymine.

\section{Protein purification}

NA26 cells carrying pTKM601 were grown at $30^{\circ} \mathrm{C}$ in $2.4 \mathrm{~L}$ of LB medium containing $100 \mu \mathrm{g} / \mathrm{mL}$ ampicillin and $50 \mu \mathrm{g} / \mathrm{mL}$ thymine. When the absorbance at $660 \mathrm{~nm}\left(A_{660}\right)$ of the culture reached $0.4-0.6$, arabinose $(0.5 \%)$ was added, and cells were incubated for an additional $2 \mathrm{~h}$. The cells were collected by centrifugation, resuspended in buffer E $(40 \mathrm{mM}$ HEPES-KOH at $\mathrm{pH}$ 7.6, $0.1 \mathrm{mM}$ EDTA, 20\% sucrose, and $10 \mathrm{mM} \beta$-mercaptoethanol) containing $250 \mathrm{mM} \mathrm{KCl}$, frozen in the presence of $20 \mathrm{mM}$ spermidine- $\mathrm{HCl}$ and $0.3 \mathrm{mg} / \mathrm{mL}$ lysozyme, and then thawed. The supernatants were collected by centrifugation and applied to a Ni-NTA agarose column (1-mL bed volume; Qiagen). After the column was washed, hisDiaA was eluted in buffer E containing $100 \mathrm{mM}$ imidazole and $250 \mathrm{mM} \mathrm{KCl}$. The eluted fractions were further purified on a Superdex 75 HR 10/30 column equilibrated with buffer C (40 mM HEPES-KOH at $\mathrm{pH} 7.6,15 \%$ [w/v] glycerol, $1 \mathrm{mM}$ EDTA, and $2 \mathrm{mM}$ dithiotheritol) containing $500 \mathrm{mM} \mathrm{KCl}$, using a flow rate of $3 \mathrm{~mL} / \mathrm{h}$. Protein concentrations were determined using a Bradford assay kit (Bio-Rad). DiaA mutant proteins were purified in a similar manner.

\section{Crystallization and structural analysis of DiaA}

DNA fragments encoding DiaA or DnaA domains I-II were inserted into pET28a (Novagen) at the NheI-BamHI sites or the NheI-HindIII sites, respectively. The resultant plasmids were introduced into $E$. coli BL21 cells to generate N-terminal $\mathrm{His}_{6}-$ tagged fusions of DiaA and DnaA domains I-II. Cells were grown at $37^{\circ} \mathrm{C}$ to an optical density at $600 \mathrm{~nm}\left(A_{600}\right)$ of 0.6 , and IPTG $(1 \mathrm{mM})$ was added to induce protein expression. After $3 \mathrm{~h}$, the cells were harvested, resuspended in buffer A $(25 \mathrm{mM}$ HEPES-KOH at pH 7.6, $200 \mathrm{mM} \mathrm{KCl}$, and $10 \%$ glycerol), disrupted by sonication, and centrifuged at $27,000 \mathrm{~g}$ for $15 \mathrm{~min}$ at $4^{\circ} \mathrm{C}$. Supernatants containing either $\mathrm{His}_{6}$-tagged DiaA or DnaA domains I-II were separately loaded onto a HisTrap column (5 $\mathrm{mL}$; Amersham Biosciences), and the column was washed with buffer A containing $20 \mathrm{mM}$ imidazole. Proteins were eluted in a 
15-column volume linear gradient of $20-500 \mathrm{mM}$ imidazole in buffer $\mathrm{A}$. The fractions containing his-tagged protein were dialyzed against buffer A, and Thrombin protease (Amersham Biosciences) was added to cleave the His tag. To purify DiaA, fractions were loaded onto a HiTrap Q column $(5 \mathrm{~mL}$; Amersham Biosciences) equilibrated with buffer A. The column was washed with 10-column volumes of buffer $\mathrm{A}$, and the protein was eluted with a 10-column volume linear gradient of 0.2-0.6 $\mathrm{M} \mathrm{KCl}$ in buffer A, and DiaA peak fractions were collected. To purify DnaA domains I-II, fractions were loaded onto a Hiload 26/60 Superdex 75 column (Amersham Biosciences) equilibrated with buffer B (25 mM HEPES-KOH at $\mathrm{pH} 7.6,400 \mathrm{mM}$ $\mathrm{KCl}$, and $10 \%$ glycerol). The purified DiaA and DnaA domains I-II were mixed at a 2:1 molar ratio, and the mixture was concentrated to $12 \mathrm{mg} / \mathrm{mL}$, with simultaneous exchange of buffer. Crystals of the DiaA-DnaA domains I-II mixture were obtained by the hanging drop method after mixing equal volumes of protein solution and reservoir solution containing $0.1 \mathrm{M}$ sodium citrate ( $\mathrm{pH} 5.4), 25 \%$ dioxane, and $20 \%$ polyethylene glycol 4000. Crystals were briefly suspended in a cryoprotectant buffer containing $0.1 \mathrm{M}$ Na-cacodylate $(\mathrm{pH} 6.0), 25 \%$ dioxane, $20 \%$ polyethylene glycol 4000, and 5\% 2-methyl-2,4-pentanediol, and flash-frozen in a stream of $\mathrm{N}_{2}$ gas (100 K). The data set was collected up to $1.85 \AA$ resolution from beamline BL44B2 at SPring- 8 in Harima. The crystals obtained belong to the orthorhombic space group $\mathrm{C} 2$, with unit cell constants of $a=71.36 \AA$, $b=81.21 \AA$, and $c=67.41 \AA$. They contained two DiaA molecules per asymmetric unit. The crystal structure was solved by molecular replacement using the MolRep program and the crystal structure of $P$. aeruginosa phosphoheptose isomerase $\mathrm{GmhA}$ (PDB ID: 1X92) as a search model. The structure was refined using the CNS program, and the final $R_{\text {free }}$ and $R_{\text {work }}$ were $21.4 \%$ and $18.3 \%$, respectively.

\section{Glycerol gradient ultracentrifugation}

The indicated amounts of hisDiaA samples were layered on a 2.2 -mL glycerol gradient $(15 \%-30 \%)$ in buffer C. A parallel gradient was used for size marker proteins. After centrifugation at $55,000 \mathrm{rpm}$ for $16 \mathrm{~h}$ in a Beckman TLS55 rotor at $2^{\circ} \mathrm{C}$, fractions $(200 \mu \mathrm{L})$ were collected from the bottom of the tube. Proteins used for size markers were $\beta$-amylase $(200 \mathrm{kDa})$, bovine serum albumin $(66 \mathrm{kDa})$, and carbonic anhydrase $(29 \mathrm{kDa})$.

\section{Flow cytometry analysis}

Flow cytometry was performed as described previously with minor modifications (Kawakami et al. 2006). Briefly, cells were exponentially grown for $\sim 10$ generations at $30^{\circ} \mathrm{C}$, followed by incubation in the presence of rifampicin $(300 \mu \mathrm{g} / \mathrm{mL})$ and cephalexin $(10 \mu \mathrm{g} / \mathrm{mL})$ for an additional $4 \mathrm{~h}$. Cells were collected in cold $70 \%$ ethanol, washed, and resuspended in cold buffer containing $10 \mathrm{mM}$ Tris- $\mathrm{HCl}(\mathrm{pH}$ 7.5), $10 \mathrm{mM}$ magnesium sulfate, and $2 \mu \mathrm{M}$ SYTOX green (Invtrogen), then analyzed using a FACScalibur flow cytometry system (Becton Dickinson). KA413 (dnaA46) cells incubated at $42^{\circ} \mathrm{C}$ and containing one or two chromosomes per cell were used to calibrate for chromosome number.

\section{In vitro minichromosomal DNA replication}

The in vitro DnaA-complementation system using minichromosome M13KEW101 and a crude protein extract (TK24 fraction II) was previously described (Ishida et al. 2004).
Pull-down assays for hisDiaA-bioDnaA complex formation and binding of non-tagged DnaA

hisDiaA-bioDnaA complexes were isolated as previously described (Ishida et al. 2004). Briefly, hisDiaA was incubated for 5 min on ice in $50 \mu \mathrm{L}$ of buffer $\mathrm{F}(50 \mathrm{mM}$ HEPES-KOH at $\mathrm{pH}$ 7.6, $1 \mathrm{mM}$ EDTA, $2 \mathrm{mM}$ DTT, and $20 \%$ sucrose) containing $100 \mathrm{mM}$ $\mathrm{KCl}, 100 \mu \mathrm{g} / \mathrm{mL}$ bovine serum albumin, $10 \mu \mathrm{M}$ ATP, and $10 \mathrm{mM}$ magnesium acetate in the presence or absence of bioDnaA. Proteins were incubated for an additional $1 \mathrm{~h}$ at $4^{\circ} \mathrm{C}$ in the presence of streptavidin-conjugated magnetic beads (Promega). The beads were collected and washed, and bound material was isolated using magnetic force, then incubated in standard SDS sample buffer $(10 \mu \mathrm{L})$ to dissociate bound hisDiaA. Proteins were then incubated in boiling water to dissociate bound bioDnaA. To analyze non-tagged DnaA binding, hisDiaA-bioDnaA complexes bound to streptavidin beads were isolated, washed, and incubated for $30 \mathrm{~min}$ at $4^{\circ} \mathrm{C}$ in the presence of non-tagged DnaA.

\section{Pull-down assay for hisDiaA-DnaA complexes}

Wild-type hisDiaA was incubated for $15 \mathrm{~min}$ on ice in buffer NP $(12.5 \mu \mathrm{L})$ containing $5 \mathrm{mM}$ Tris- $\mathrm{HCl}(\mathrm{pH} 7.5), 10 \%$ glycerol, 8 $\mathrm{mM}$ 2-mercaptoethanol, 0.01\% Brij-58, $8 \mathrm{mM}$ magnesium acetate, $100 \mathrm{mM} \mathrm{KCl}$, and $0.1 \mathrm{mM}$ ATP in the presence of DnaA. Proteins were incubated for an additional $5 \mathrm{~min}$ on ice in the presence of Dynabeads TALON (20 $\mu \mathrm{g}$, Invitrogen) and $10 \mathrm{mM}$ imidazole in the same buffer $(25 \mu \mathrm{L})$ as above. The beads and bound materials were collected and washed in buffer NP $(2.5 \mu \mathrm{L})$ containing $30 \mathrm{mM}$ imidazole. Proteins were eluted in buffer NP $(8 \mu \mathrm{L})$ containing $60 \mathrm{mM}$ imidazole.

\section{Pull-down assay using bio-oriC}

A bio-oriC fragment (419 bp) was amplified by PCR using an oriC plasmid, M13KEW101, a $5^{\prime}$-biotinylated primer $\left(5^{\prime}\right.$-CCC GGGCCGTGGATTCTAC-3'), and an unmodified primer (5'GAAGATCAACATTCTTGATCACG-3'). DnaA, hisDiaA, and bio-oriC (100 fmol) were incubated for $30 \mathrm{~min}$ on ice in buffer $\mathrm{F}$ $(50 \mu \mathrm{L})$ containing $100 \mathrm{mM} \mathrm{KCl}, 100 \mu \mathrm{g} / \mathrm{mL}$ bovine serum albumin, $1 \mathrm{mM}$ ATP, and $10 \mathrm{mM}$ magnesium acetate. The mixture was further incubated for $1 \mathrm{~h}$ at $4^{\circ} \mathrm{C}$ with gentle rotation in the presence of streptavidin-beads (Promega) equilibrated in the same buffer $(50 \mu \mathrm{L})$ as above. The beads and bound material were collected, washed in buffer $\mathrm{F}(50 \mu \mathrm{L})$ containing $100 \mathrm{mM}$ $\mathrm{KCl}, 1 \mathrm{mM}$ ATP, and $10 \mathrm{mM}$ magnesium acetate $(50 \mu \mathrm{L})$, resuspended in $10 \mu \mathrm{L}$ of standard SDS sample buffer, and analyzed by $13 \%$ SDS-PAGE.

\section{Gel-mobility retardation assay}

The gel-mobility assay was performed as previously described (Kawakami et al. 2006; Ozaki et al. 2006). Briefly, DnaA was incubated for $20 \mathrm{~min}$ on ice in buffer $(10 \mu \mathrm{L})$ containing $2 \mathrm{mM}$ ATP and the 15-mer DnaA R1 box (2.5 pmol) in the presence or absence of hisDiaA (20 pmol). The samples were analyzed by $8 \%$ PAGE, followed by Gel-star (Cambrex) staining, then quantified by densitometry. Protein-free DNA was also quantified and is presented as a percentile.

Open complex formation assay using P1 nuclease

The P1 nuclease assay was performed as previously described (Kawakami et al. 2006; Ozaki et al. 2006). Briefly, the indicated amounts of hisDiaA were incubated for $3 \mathrm{~min}$ at $38^{\circ} \mathrm{C}$ in buffer 
$(50 \mu \mathrm{L})$ containing $5 \mathrm{mM}$ ATP, $16 \mathrm{ng}$ of $\mathrm{HU}$ protein, M13KEW101 oriC plasmid (320 ng; $61 \mathrm{fmol})$, and ATP-DnaA (0 or $1.2 \mathrm{pmol})$, followed by incubation for $200 \mathrm{sec}$ in the presence of P1 nuclease (10 units). DNA was purified, and one-fourth of each sample was digested with AlwNI, then analyzed by $1 \%$ agarose gel electrophoresis and ethidium bromide staining. The DNA fragments were quantified by densitometry.

\section{DNase I footprinting analysis}

DNase I footprinting analysis was performed as described previously (Kawakami et al. 2005). Briefly, the indicated amounts of DnaA and hisDiaA were incubated for $10 \mathrm{~min}$ at $30^{\circ} \mathrm{C}$ in buffer $(10 \mu \mathrm{L})$ containing $25 \mathrm{mM}$ HEPES-KOH $(\mathrm{pH} 7.6), 5 \mathrm{mM}$ calcium acetate, $2.8 \mathrm{mM}$ magnesium acetate, $35 \mathrm{mM}$ ammonium sulfate, $23 \mathrm{mM}$ potassium acetate, $4 \mathrm{mM}$ dithiothreitol, $10 \%(\mathrm{v} / \mathrm{v})$ glycerol, $0.2 \%$ Triton $\mathrm{X}-100,0.5 \mathrm{mg} / \mathrm{mL}$ bovine serum albumin, $14 \mu \mathrm{g} / \mathrm{mL}$ poly(dA-dT)-(dA-dT), $14 \mu \mathrm{g} / \mathrm{mL}$ poly(dI-dC)(dI-dC), 3 mM ATP, and ${ }^{32} \mathrm{P}$-end-labeled oriC fragment $(5.5 \mathrm{ng}$ ), followed by incubation for $4 \mathrm{~min}$ at the same temperature in the presence of DNase I (0.63 mU). DNA was then purified and analyzed by $5 \%$ sequencing gel electrophoresis and analyzed using a BAS-2500 Bio-imaging analyzer (Fuji Film).

\section{Accession number}

Atomic coordinates and structure factor amplitudes of the $E$. coli DiaA have been deposited in the Protein Data Bank under accession code 2 YVA.

\section{Acknowledgments}

This study was supported mainly by Grants-in-Aid from the Ministry of Education, Culture, Sports, Science and Technology of Japan, and the RIKEN Structural Genomics/Protromics Initiative (RSGI) the National Project on Protein Structural and Functional Analyses.

\section{References}

Abe, Y., Jo, T., Matsuda, Y., Matsunaga, C., Katayama, T., and Ueda, T. 2007. Structure and function of DnaA N-terminal domains: Specific sites and mechanisms in inter-DnaA interaction and in DnaB helicase loading on oriC. J. Biol. Chem. 282: 17816-17827.

Arunkumar, A.I., Stauffer, M.E., Bochkareva, E., Bochkarev, A., and Chazin, W.J. 2003. Independent and coordinated functions of replication protein A tandem high-affinity singlestranded DNA binding domains. J. Biol. Chem. 278: $41077-$ 41082.

Bateman, A. 1999. The SIS domain; a phosphosugar-binding domain. Trends Biochem. Sci. 24: 94-95.

Bleasing, F., Weigel, C., Welzeck, M., and Messer, W. 2000. Analysis of the DNA-binding domain of Escherichia coli DnaA protein. Mol. Microbiol. 36: 557-569.

Botchan, M. 2007. A switch for S phase. Nature 445: 272-274.

Brooke, J.S. and Valvano, M.A. 1996. Molecular cloning of the Haemophilus influenzae gmhA (1cpA) gene encoding a phosphoheptose isomerase required for lipooligosaccharide biosynthesis. J. Bacteriol. 178: 3339-3341.

Burz, D.S., Beckett, D., Benson, N., and Ackers, G.K. 1994. Selfassembly of bacteriophage $\lambda$ cI repressor: Effects of singlesite mutations on the monomer-dimer equilibrium. Biochemistry 33: 8399-8405.

Erzberger, J.P., Pirruccello, M.M., and Berger, J.M. 2002. The structure of bacterial DnaA: Implications for general mechanisms underlying DNA replication initiation. EMBO J. 21:
$4763-4773$.

Erzberger, J.P., Mott, M.L., and Berger, J.M. 2006. Structural basis for ATP-dependent DnaA assembly and replicationorigin remodeling. Nat. Struct. Mol. Biol. 13: 676-683.

Fujikawa, N., Kurumizaka, H., Nureki, O., Terada, T., Shirouzu, M., Katayama, T., and Yokoyama, S. 2003. Structural basis of replication origin recognition by the DnaA protein. Nucleic Acids Res. 31: 2077-2086.

Guo, L., Katayama, T., Seyama, Y., Sekimizu, K., and Miki, T. 1999. Isolation and characterization of novel cold-sensitive dnaA mutants of Escherichia coli. FEMS Microbiol. Lett. 176: $357-366$.

Hanson, C.J., Bootman, M.D., and Roderick, H.L. 2004. Cell signalling: IP3 receptors channel calcium into cell death. Curr. Biol. 14: R933-R935.

Ishida, T., Akimitsu, N., Kashioka, T., Hatano, M., Kubota, T., Ogata, Y., Sekimizu, K., and Katayama, T. 2004. DiaA, a novel DnaA-binding protein, ensures the timely initiation of Escherichia coli chromosome replication. J. Biol. Chem. 279: 45546-45555.

Kaguni, J.M. 2006. DnaA: Controlling the initiation of bacterial DNA replication and more. Annu. Rev. Microbiol. 60: 351375.

Katayama, T. and Crooke, E. 1995. DnaA protein is sensitive to a soluble factor and is specifically inactivated for initiation of in vitro replication of the Escherichia coli minichromosome. J. Biol. Chem. 270: 9265-9271.

Katayama, T., Kubota, T., Kurokawa, K., Crooke, E., and Sekimizu, K. 1998. The initiator function of DnaA protein is negatively regulated by the sliding clamp of the E. coli chromosomal replicase. Cell 94: 61-71.

Kato, J. and Katayama, T. 2001. Hda, a novel DnaA-related protein, regulates the replication cycle in Escherichia coli. EMBO I. 20: 4253-4262.

Kawakami, H., Keyamura, K., and Katayama, T. 2005. Formation of an ATP-DnaA-specific initiation complex requires DnaA arginine 285, a conserved motif in the AAA+ protein family. J. Biol. Chem. 280: 27420-27430.

Kawakami, H., Ozaki, S., Suzuki, S., Nakamura, K., Senriuchi, T., Su'etsugu, M., Fujimitsu, K., and Katayama, T. 2006. The exceptionally tight affinity of DnaA for ATP/ADP requires a unique aspartic acid residue in the AAA+ sensor 1 motif. Mol. Microbiol. 62: 1310-1324.

Kim, Y.K., Lee, K.J., Jeon, H., and Yu, Y.G. 2006. Protein kinase CK2 is inhibited by human nucleolar phosphoprotein p140 in an inositol hexakisphosphate-dependent manner. J. Biol. Chem. 281: 36752-36757.

Kurokawa, K., Nishida, S., Emoto, A., Sekimizu, K., and Katayama, T. 1999. Replication cycle-coordinated change of the adenine nucleotide-bound forms of DnaA protein in Escherichia coli. EMBO J. 18: 6642-6652.

Margulies, C. and Kaguni, J.M. 1996. Ordered and sequential binding of DnaA protein to oriC, the chromosomal origin of Escherichia coli. J. Biol. Chem. 271: 17035-17040.

McGarry, K.C., Ryan, V.T., Grimwade, J.E., and Leonard, A.C. 2004. Two discriminatory binding sites in the Escherichia coli replication origin are required for DNA strand opening by initiator DnaA-ATP. Proc. Natl. Acad. Sci. 101: 28112816.

Messer, W. 2002. The bacterial replication initiator DnaA DnaA and oriC, the bacterial mode to initiate DNA replication. FEMS Microbiol. Rev. 26: 355-374.

Nishida, S., Fujimitsu, K., Sekimizu, K., Ohmura, T., Ueda, T., and Katayama, T. 2002. A nucleotide switch in the Escherichia coli DnaA protein initiates chromosomal replication: Evidence from a mutant DnaA protein defective in 
regulatory ATP hydrolysis in vitro and in vivo. J. Biol. Chem. 277: 14986-14995.

Nordström, K. 2006. Plasmid R1-replication and its control. Plasmid 55: 1-26.

O'Donnell, M. 2006. Replisome architecture and dynamics in Escherichia coli. J. Biol. Chem. 281: 10653-10656.

Ozaki, S., Fujimitsu, K., Kurumizaka, H., and Katayama, T. 2006. The DnaA homolog of the hyperthermophilic eubacterium Thermotoga maritima forms an open complex with a minimal 149-bp origin region in an ATP-dependent manner. Genes Cells 11: 425-438.

Slater, S., Wold, S., Lu, M., Boye, E., Skarstad, K., and Kleckner, N. 1995. E. coli SeqA protein binds oriC in two different methyl-modulated reactions appropriate to its roles in DNA replication initiation and origin sequestration. Cell 82: 927936.

Speck, C. and Messer, W. 2001. Mechanism of origin unwinding: Sequential binding of DnaA to double- and singlestranded DNA. EMBO J. 20: 1469-1476.

Stauffer, M.E. and Chazin, W.J. 2004. Structural mechanisms of DNA replication, repair, and recombination. J. Biol. Chem. 279: 30915-30918.

Stillman, B. 2005. Origin recognition and the chromosome cycle. FEBS Lett. 579: 877-884.

Sutton, M.D. and Kaguni, J.M. 1997. Threonine 435 of Escherichia coli DnaA protein confers sequence-specific DNA binding activity. J. Biol. Chem. 272: 23017-23024.

Tan, L. and Darby, C. 2006. Yersinia pestis YrbH is a multifunctional protein required for both 3-deoxy-D-manno-oct2-ulosonic acid biosynthesis and biofilm formation. Mol. Microbiol. 61: 861-870.

Weigel, C., Schmidt, A., Rückert, B., Lurz, R., and Messer, W. 1997. DnaA protein binding to individual DnaA boxes in the Escherichia coli replication origin, oriC. EMBO J. 16: 65746583.

Zhou, H.X. 2001. Single-chain versus dimeric protein folding: Thermodynamic and kinetic consequences of covalent linkage. J. Am. Chem. Soc. 123: 6730-6731.

Zyskind, J.W. and Smith, D.W. 1992. DNA replication, the bacterial cell cycle, and cell growth. Cell 69: 5-8. 


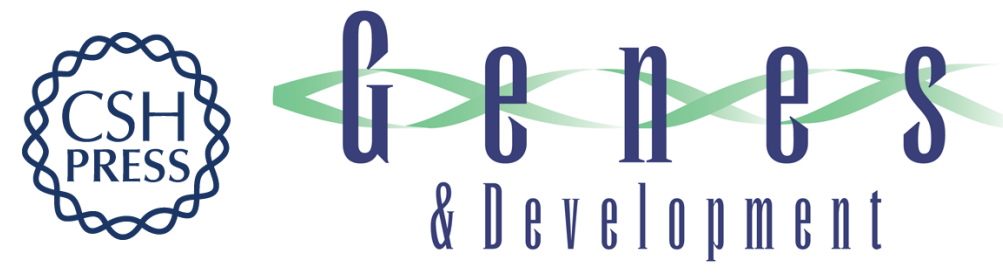

\section{The interaction of DiaA and DnaA regulates the replication cycle in $E$. coli by directly promoting ATPDnaA-specific initiation complexes}

Kenji Keyamura, Norie Fujikawa, Takuma Ishida, et al.

Genes Dev. 2007, 21:

Access the most recent version at doi:10.1101/gad.1561207

Supplemental http://genesdev.cshlp.org/content/suppl/2007/08/06/21.16.2083.DC1
Material

References This article cites 37 articles, 18 of which can be accessed free at:

http://genesdev.cshlp.org/content/21/16/2083.full.html\#ref-list-1

License

Email Alerting Receive free email alerts when new articles cite this article - sign up in the box at the top

Service right corner of the article or click here.

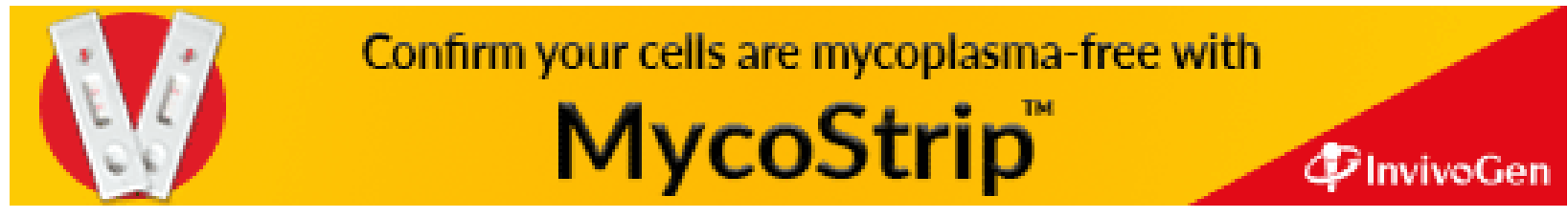

